Mon. Not. R. Astron. Soc. 000, 000-000 (0000) Printed 27 May 2019 (MN LATEX style file v2.2)

\title{
A Massive Cluster at $z=0.288$ Caught in the Process of Formation: The Case of Abell 959
}

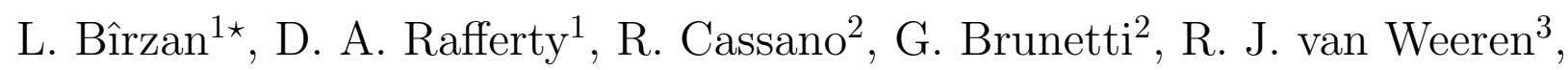
M. Brüggen ${ }^{1}$, H. T. Intema ${ }^{3,4}$, F. de Gasperin ${ }^{1}$, F. Andrade-Santos ${ }^{5}$, A. Botteon ${ }^{2,6}$, H. J. A. Röttgering ${ }^{3}$, T. W. Shimwell ${ }^{7}$

${ }^{1}$ Hamburger Sternwarte, Universität Hamburg, Gojenbergsweg 112, 21029, Hamburg, Germany

${ }^{2}$ INA F-Istituto di Radioastronomia, via P. Gobetti, 101, I-40129, Bologna, Italy

${ }^{3}$ Leiden Observatory, Leiden University, Oort Gebouw, P.O. Box 9513, 2300 RA Leiden, The Netherlands

${ }^{4}$ International Centre for Radio Astronomy Research - Curtin University, GPO Box U1987, Perth, WA 6845, Australia

${ }^{5}$ Harvard-Smithsonian Center for Astrophysics, 60 Garden Street, Cambridge, MA 02138, USA

${ }^{6}$ Dipartimento di Fisica e Astronomia, Universit di Bologna, via P. Gobetti 93/2, I-40129 Bologna, Italy

7 Netherlands Institute for Radio Astronomy (ASTRON), P.O. Box 2, 7990 AA Dwingeloo, The Netherlands

27 May 2019

\begin{abstract}
The largest galaxy clusters are observed still to be forming through major clustercluster mergers, often showing observational signatures such as radio relics and giant radio haloes. Using LOFAR Two-meter Sky Survey data, we present new detections of both a radio halo (with a spectral index of $\alpha_{143}^{1400}=1.48_{-0.23}^{+0.06}$ ) and a likely radio relic in Abell 959, a massive cluster at a redshift of $z=0.288$. Using a sample of clusters with giant radio haloes from the literature ( 80 in total), we show that the radio halo in A959 lies reasonably well on the scaling relations between the thermal and non-thermal power of the system. Additionally, we find evidence that steep-spectrum haloes tend to reside in clusters with high X-ray luminosities relative to those expected from cluster $L M$ scaling relations, indicating that such systems may preferentially lie at an earlier stage of the merger, consistent with the theory that some steep-spectrum haloes result from low-turbulence mergers. Lastly, we find that halo systems containing radio relics tend to lie at lower X-ray luminosities, relative to those expected from cluster $L M$ scaling relations, for a given halo radio power than those without relics, suggesting that the presence of relics indicates a later stage of the merger, in line with simulations.
\end{abstract}

Key words: galaxies: clusters: individual: A959 - radio continuum: galaxies - cosmology: large-scale structure of Universe - X-rays: galaxies: clusters - intracluster medium .

\section{INTRODUCTION}

In the present-day Universe, many clusters are still forming through hierarchical processes and major merger events with neighboring clusters (e.g., Press \& Schechter 1974; Springel et al. 2006; Kravtsov \& Borgani 2012). On smaller scales, non-gravitational processes, such as radiative cooling, supernova heating, and feedback from active galactic nuclei (AGN), are also important (Benson et al. 2003; Scannapieco \& Oh 2004; Bîrzan et al. 2004; Voit et al. 2005; McNamara \& Nulsen 2007; Fabian 2012; Alexander \& Hickox 2012). As such, clusters of galaxies have wide-ranging astrophysical applications. For example, they can be used to constrain the cosmological parameters (Allen et al. 2011) and to provide constraints on the properties of dark matter (Markevitch et al. 2004; Clowe et al. 2004, 2006; Harvey et al. 2015).

All of the processes important to the formation of clusters dissipate energy into the intra-cluster medium (ICM) through shocks: e.g., accretion shocks, merger shocks, AGN related shocks, or ICM bulk motion shocks (see the reviews of Brüggen et al. 2012; Brunetti \& Jones 2014). Observationally, merger shocks have been detected in Chandra Xray and XMM-Newton observations of a small number of merging clusters (e.g., Bullet Cluster, A520, A521, A2146, A3667, A754, El Gordo, A665, A2219, and A2744; Markevitch et al. 2002; Shimwell et al. 2015; Markevitch et al. 2005; Giacintucci et al. 2008; Bourdin et al. 2013; Russell et al. 2010; Finoguenov et al. 2010; Sarazin et al. 2016; Macario 
et al. 2011; Botteon et al. 2016; Dasadia et al. 2016; Canning et al. 2017; Eckert et al. 2016; Pearce et al. 2017) with modest Mach numbers of $M=1.5-3$ and, in radio images, in the form of large-scale, diffuse emission associated with the shocks (e.g., Shimwell et al. 2014; Botteon et al. 2016; Vacca et al. 2014; Giacintucci et al. 2008; Golovich et al. 2018). Such radio structures, known as radio relics (or radio shocks, see the review of van Weeren et al. 2019), have polarized emission resulting from ordered magnetic fields aligned by the shock. The favored mechanism for the relic creation is the acceleration of electrons by diffuse shock acceleration (DSA), where the electrons can either come from the thermal pool (e.g., Ensslin et al. 1998; Pfrommer et al. 2008) or be mildly relativistic cosmic rays (CRe; e.g., fossil electrons from previous AGN or merger activity, Markevitch et al. 2005; Kang et al. 2012; Pinzke et al. 2013; van Weeren et al. 2017a).

In addition to radio relics, a number of luminous Xray clusters show diffuse, cluster-scale radio emission known as giant radio haloes (Venturi et al. 2007, 2008; Kale et al. $2013,2015)$. The giant radio haloes (RHs) are thought to form from the post-merger turbulence of seed suprathermal CRe (e.g.,turbulent re-acceleration model; Brunetti et al. 2001; Petrosian 2001; Cassano et al. 2006, 2007; Brunetti et al. 2009; Cassano et al. 2010a; Brunetti \& Lazarian 2011; Donnert et al. 2013; Brunetti \& Lazarian 2016; Pinzke et al. 2017; Eckert et al. 2017; Brunetti et al. 2017). In support of this scenario, there exists a connection between the presence of a halo and the presence of merging activity, with the radio luminosity of the halo $\left(P_{1.4 \mathrm{GHz}}\right)$ correlating with the X-ray luminosity of the cluster $\left(L_{\mathrm{X}}\right)$, the mass of the cluster $(M)$ or the Sunyaev-Zel'dovich signal $\left(Y_{\mathrm{SZ}}\right)$, albeit with large scatter (Cassano et al. 2007; Brunetti et al. 2007, 2009; Cassano et al. 2010b; Basu 2012; Cassano et al. 2013; Kale et al. 2015).

Giant radio haloes ( $\mathrm{RHs}$ ) are generally observed in clusters whose X-ray gas has a long central cooling time, $t_{\text {cool }}>10^{9}$ yr. These clusters are known as non-cooling flow clusters $(\mathrm{NCF})$, whereas those with shorter cooling times are known as cooling flow $(\mathrm{CF})$ or cool-core $(\mathrm{CC})$ clusters. In NCFs, the radio power of the central radio source is typically below $L_{1.4 \mathrm{GHz}}<2.5 \times 10^{30} \mathrm{erg} \mathrm{s}^{-1} \mathrm{~Hz}^{-1}$ (Bîrzan et al. 2012). However, there are a few systems known to have a short central cooling time and to possess a giant radio halo (e.g., EL Gordo, H1821+643; Bîrzan et al. 2017; Lindner et al. 2014; Russell et al. 2010; Bonafede et al. 2014b). There are also systems which are seen to have an intermediate (or large) cooling time and a two-component $\mathrm{RH}$ : a $\mathrm{RH}$ plus a radio mini-halo (e.g.; RXJ1347.5-1145, A2319, A2142, RXJ1720.1+2638, PSZ1G139.61+24; Ferrari et al. 2011; Storm et al. 2015; Venturi et al. 2017; Savini et al. 2018, 2019). The details of how CF and NCF systems form and relate to each other are still not fully understood (e.g., Poole et al. 2008; Burns et al. 2008; Parrish et al. 2010; Pfrommer et al. 2012; Rasia et al. 2015; Hahn et al. 2017; Medezinski et al. 2017), and the observational bias of X-ray selected samples complicates the issue (Rossetti et al. 2017; Andrade-Santos et al. 2017).

Giant RHs and radio relics are found in a significant percentage of massive clusters (e.g., 23\% for EGRHS, Kale et al. 2013). Therefore, to date, most radio campaigns searching for such RHs have focused on luminous X-ray clus- ters $\left(L_{X}>5 \times 10^{44} \mathrm{erg} \mathrm{s}^{-1}\right)$, typically between redshifts of 0.2-0.4 (e.g., the Extended Giant Meterwave Radio Telescope -GMRT- Radio Halo Survey, EGRHS, Venturi et al. 2007, 2008, 2013; Kale et al. 2013, 2015). However, semianalytical models and cosmological simulations have predicted that sensitive low-frequency radio observations, such as those made with LOFAR at $\sim 150 \mathrm{MHz}$, should commonly find haloes in less massive systems (Cassano et al. 2006, 2010a, 2012; Zandanel et al. 2014) as well as thousands of more radio relics (Hoeft et al. 2011; Nuza et al. 2012).

Abell 959 (hereafter A959), the subject of this study, is situated at a redshift of $z=0.288$, has a mass of $M_{\mathrm{SZ} 500}=(5.08 \pm 0.47) \times 10^{15} \mathrm{M}_{\odot} \quad$ (Planck Collaboration et al. 2014) and multiple galaxy concentrations (Boschin et al. 2009). Multiple mass concentrations in A959 were identified from a weak gravitational lensing analysis (Dahle et al. 2002, 2003). Among these concentrations is a putative dark mass clump (WL 1017.3+5931) that is not associated with a known galaxy concentration or X-ray gas clump. Furthermore, Boschin et al. (2009), using spectroscopic observations, found a redshift of $z=0.288$, lower than the value of $z=0.353$ used previously in the literature (see also Irgens et al. 2002). They concluded that the cluster is in an early, dynamical stage of formation and might be forming along two main directions of mass accretion. Diffuse radio emission in A959 was reported in Cooray et al. (1998) and Owen et al. (1999), and the latter found a flux density at $1.4 \mathrm{GHz}$ of $3 \mathrm{mJy}$ and a size of $0.8 \mathrm{Mpc}$. However, A959 has not been studied at lower frequencies or in detail in X-rays up to now.

In this paper we present the results of a multiwavelength study of A959. We use LOFAR data to study the radio emission and X-ray data from the XMM-Newton and Chandra X-ray observatories to measure the cluster properties and to place constraints on gas mass fraction of the putative dark mass clump (WL 1017.3+5931). Using a large sample drawn from the literature, we place A959 in context with other RH systems and we investigate the evolution of the X-ray and radio properties of $\mathrm{RH}$ clusters. We adopt $H_{0}=70 \mathrm{~km} \mathrm{~s}^{-1} \mathrm{Mpc}^{-1}, \Omega_{\Lambda}=0.7$, and $\Omega_{\mathrm{M}}=0.3$ throughout.

\section{DATA ANALYSIS}

\subsection{LOFAR Data}

A959 was observed with the High-Band Array (HBA) of LOFAR at frequencies of $120-170 \mathrm{MHz}$ on 25-04-2015 for 8 hours as part of observing program LC3_008 (taken as part of LoTSS, the LOFAR Two-meter Sky Survey; Shimwell et al. 2017). A 10-minute observation of a calibrator, 3C196, was made immediately preceding the A959 observation and is used to set the overall flux (Scaife \& Heald 2012) and to remove instrumental phase effects from the visibility data. Preprocessing of the data from both observations included flagging of radio-frequency interference (RFI) and averaging in time and frequency (to reduce the raw visibility data to a manageable size). These preprocessed data were obtained from the LOFAR long-term archive and further processed 
using the PREFACTOR ${ }^{1}$ and FACTOR pipelines ${ }^{2}$ to calibrate and image the data using the facet-calibration scheme described in van Weeren et al. (2016b). Version 2.0.2 of PREFACTOR and version 1.3 of FACTOR were used.

The PREFACTOR pipeline first derives the bandpass calibration and corrects for instrumental phase effects using the 3C196 calibrator observation. For each station, amplitude and phase corrections, plus an additional term that tracks the rotation angle between the XX and YY phases, were solved for each of the XX and YY polarizations every 4 seconds and $48.8 \mathrm{kHz}$. The model of 3C196 of XX was used for the calibration. For each time slot and station, the phase solutions are then fit with a model that is comprised of a clock term that scales with the frequency, $\nu$, a differential total electron content (dTEC) term that scales as $\nu^{-1}$, and an offset term that is constant in frequency. The clock and offset solutions are then transferred, along with the amplitudes, to the target data. In this way, the direction-independent instrumental effects are corrected for.

Next, the PREFACTOR pipeline groups the data into bands of $\approx 2 \mathrm{MHz}$ each, the maximum bandwidth over which frequency-dependent effects can be largely ignored (and therefore fit with a single solution in frequency). Each of these bands is then phase calibrated using a model of the field obtained from the TIFR GMRT Sky Survey catalog (TGSS, Intema et al. 2017) and imaged. The imaging is done in two passes, with the purpose of modeling the sources in the field out to the second null of the primary beam. To this end, two images are made of each band: one at a resolution of $\sim 25^{\prime \prime}$, used to detect and model the compact emission, and one at a resolution of $\sim 75^{\prime \prime}$, used to model any diffuse, extended emission not picked up in the higher-resolution image. The lower-resolution image is made of the residual visibilities, after subtraction of the higher-resolution clean components. Components from both images are then subtracted from the uv-data to produce "source-free" datasets suitable for use in FACTOR.

After PREFACTOR was run, FACTOR was used to correct for direction-dependent effects. The main directiondependent effects in HBA LOFAR data are due to phase delays induced by the ionosphere and amplitude errors that occur due to inaccuracies in the LOFAR beam model. FACTOR corrects for these effects by faceting the field and solving for a single set of corrections for each facet. The field was divided into 45 facets, of which 12 were processed. The processed facets were those that contained very bright sources and those that neighbored on (or included) A959 (the 33 unprocessed facets contain only fainter, more distant sources that do not affect the Abell 959 facet). FACTOR was run with the default parameters. The full bandwidth was used in the imaging, resulting in an image with a frequency of 143.7 MHz and an rms noise of $103 \mu \mathrm{Jy}^{\text {beam }^{-1}}$ at the field center.

The global flux scale was checked by extracting the LOFAR flux densities of the 41 brightest unresolved sources in the processed facets and comparing them to the TGSS flux densities. We found the average ratio of LOFAR-to-TGSS flux density to be 1.05 , approximately the ratio expected

1 Available at https://github.com/lofar-astron/prefactor

2 Available at https://github.com/lofar-astron/factor given the slightly different frequencies of the images (143.7 $\mathrm{MHz}$ for LOFAR and $150 \mathrm{MHz}$ for the TGSS) and the average spectral index of radio sources $(\approx-0.8)$. We adopt a conservative systematic uncertainty of $15 \%$ on all LOFAR flux densities throughout our analysis, as done in previous LOFAR-HBA works.

Figure 1 shows two images at $143.7 \mathrm{MHz}$ : a highresolution image, with a restoring beam with a FWHM of $4.9^{\prime \prime} \times 8.3^{\prime \prime}$, and a low-resolution residual image, made after subtracting compact emission, with a restoring beam of $55^{\prime \prime} \times 60^{\prime \prime}$. The compact emission was modeled by imaging with a uv minimum of $4 \mathrm{k} \lambda$, a cut that results in emission on scales of $\gtrsim 60$ arcsec being excluded (see Figure 1 ). The resulting clean components were then subtracted from the visibilities (using the $f t$ and uvsub tasks in CASA v4.7.1) and the low-resolution residual image made by tapering the uv-data with a Gaussian taper to achieve a resolution of $\approx 40$ arcsec.

In the full-resolution image, a number of features are apparent: a source (source A) that is associated with the brightest cluster galaxy (BCG), with two lobes oriented approximately N-S; a source (source B) that is located to the north of the $\mathrm{BCG}$ and appears to be a head-tail radio galaxy; and a linear, relic-like feature (Source C) that does not appear to be clearly associated with any optical galaxy. In the low-resolution residual image, diffuse emission is seen that fills most of the region between the BCG and the relic-like source C. We will discuss these features in detail in Section 3.

\subsection{GMRT Data}

GMRT $325 \mathrm{MHz}$ observations of A959 were obtained on 06-03-2017 (project ID 31_009; PI de Gasperin). Visibilities were recorded over $33.3 \mathrm{MHz}$ of bandwidth, starting with 20 minutes on calibrator $3 \mathrm{C} 147$, then 213 minutes on A959, and finally 16 minutes on $3 \mathrm{C} 147$ again. The data were processed using the SPAM pipeline (Intema et al. 2017) in the default mode, and calibrated using $3 \mathrm{C} 147$ while adopting the flux scale from Scaife \& Heald (2012). This resulted in a final image with a central frequency of $322.7 \mathrm{MHz}$ and an rms noise of $84 \mu \mathrm{Jy}$ beam $^{-1}$ at the field center.

The resulting 322.7 MHz GMRT image is shown in Figure 2, with the sources identified in the high-resolution LOFAR image labeled. As with the LOFAR data, we searched for diffuse emission by modeling and subtracting the compact emission and imaging the residual data at lower resolution, but we did not detect any such emission. However, sources A and B are clearly detected in the GMRT image with very similar morphologies to those in the LOFAR image. Source C, the putative relic, is not detected (there is a hint of emission at its location, but its significance is low and may be a sidelobe of the bright source nearby).

\subsection{Chandra Data}

A959 was observed by the Chandra X-ray Observatory on 01-02-2016 for $7.6 \mathrm{ks}$ (ObsID 17161, VFAINT mode) with the ACIS-I instrument. The data were obtained from the Chandra data archive and were reprocessed with CIAO $4.8^{3}$ 

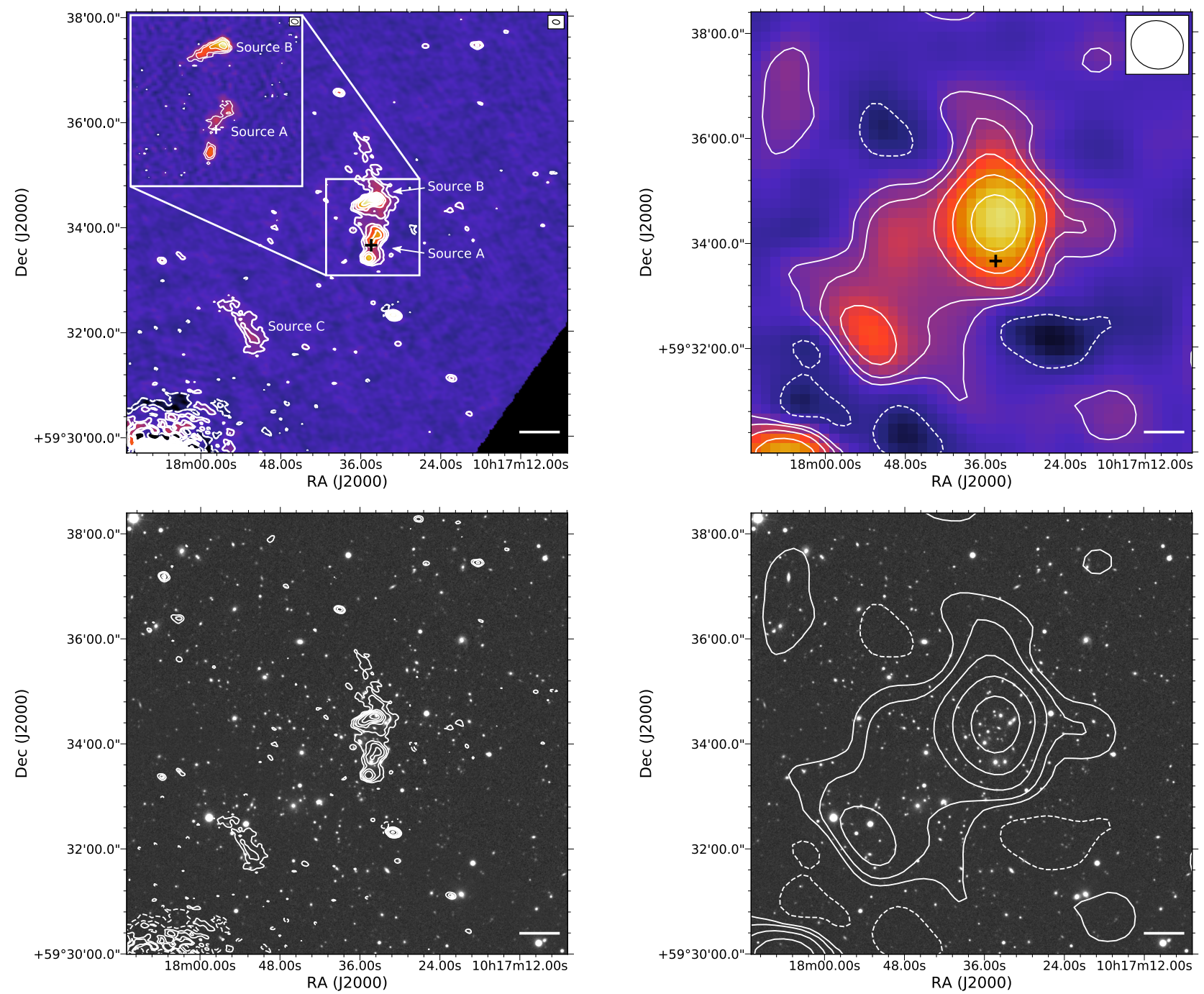

Figure 1. Top row: LOFAR images at $143.7 \mathrm{MHz}$ at high-resolution (left) and low-resolution (right) after subtraction of the compact emission. The compact emission that was subtracted is shown in the inset image in the left panel (see text for details). The restoring beam is indicated by the white ellipse in the upper right-hand corner, and the scale bar represents $200 \mathrm{kpc}$ at the redshift of A959. Contours begin at 3 times the rms noise of $118.5 \mu \mathrm{Jy}_{\text {beam }}{ }^{-1}$ and $426.7 \mu \mathrm{Jy}$ beam ${ }^{-1}$ for the high- and low-resolutions images, respectively, and increase by a factor of 2 . The first negative contour (at -3 times the rms noise) is also plotted and is denoted by the dashed lines. The cross marks the location of the BCG. Bottom row: SDSS optical $r$-band image with the contours from the high-resolution (left) and low-resolution (right) LOFAR images overlaid. In top-left panel: Source A is the central radio source associated with the BCG (see Section 3.2); source B is a tailed radio galaxy and has a flux $S_{143.7 \mathrm{MHz}}=45.3 \mathrm{mJy}$, and Source C is the candidate radio relic (see Section 3.6).

using CALDB 4.7.3 $3^{4}$. The data were corrected for known timedependent gain and charge transfer inefficiency problems, and the events files were filtered for flares using the CIAO script $l c_{-}$clean to match the filtering used during the construction of the blank-sky background files used for background subtraction. ${ }^{5}$ A total of 7.1 ks remained after filtering. The background file was normalized to the count rate of the source image in the $10-12 \mathrm{keV}$ band (after filtering). Lastly, point sources detected using the CIAO tool wavdetect were removed.

Spectra were extracted in annuli constructed to con-

\footnotetext{
4 See cxc.harvard.edu/caldb/index.html.

5 See http://asc.harvard.edu/contrib/maxim/acisbg/.
}

tain at least 500 counts each using the CIAO script specextract. For each spectrum, weighted responses were made, and a background spectrum was extracted in the same region of the CCD from the associated blank-sky background file. For the spectral fitting, XSPEC (Arnaud 1996) version 12.7.1 was used. Gas temperatures and densities were found by deprojecting these spectra using the Direct Spectral Deprojection method of Sanders \& Fabian (2007). The deprojected spectrum in each annulus was then fit in XSPEC with a single-temperature plasma model (MEKAL) absorbed by foreground absorption model (WABS), between the energies of $0.5 \mathrm{keV}$ and $7.0 \mathrm{keV}$. In this fitting, the redshift was fixed to $z=0.288$ (Boschin et al. 2009), and the foreground hydrogen column density was fixed to $N_{\mathrm{H}}=8.78 \times 10^{19} \mathrm{~cm}^{-2}$, 


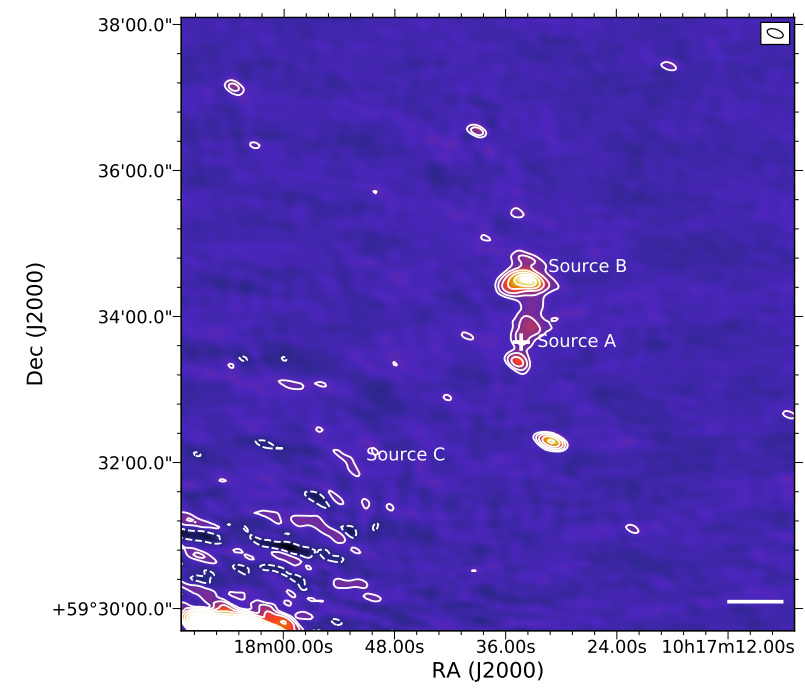

Figure 2. GMRT image at $322.7 \mathrm{MHz}$. Contours begin at 3 times the rms noise of $130.6 \mu \mathrm{Jy}_{\text {beam }}{ }^{-1}$. The restoring beam is $6.9^{\prime \prime} \times$ $13.7^{\prime \prime}$. The scale bar, symbols, and annotations are the same as in Figure 1, left.

the weighted-average Galactic value from Dickey \& Lockman (1990).

The density was calculated from the normalization of the MEKAL component, assuming $n_{\mathrm{e}}=1.2 n_{\mathrm{H}}$ (for a fully ionized gas with hydrogen and helium mass fractions of $X=0.7$ and $Y=0.28$ ). The pressure in each annulus was calculated as $P=n k T$, where we have assumed an ideal gas taking $n=2 n_{\mathrm{e}}$. The entropy is taken as $S=k T n_{\mathrm{e}}^{-2 / 3}$. The cooling time was derived from the temperature, metallicity, and density using the cooling curves of Smith et al. (2001).

We also derived the X-ray luminosity and emissionweighted temperature inside the $R_{500}$ region, defined as the region at which the mean mass density is 500 times the critical density at the cluster redshift (see Pratt et al. 2009) ${ }^{6}$. We found $R_{500}=1100 \mathrm{kpc}$ using the mass $M_{500}=(5.08 \pm 0.47) \times$ $10^{14} \mathrm{M}_{\odot}$, derived from the SZ signal $Y_{\mathrm{SZ}}$ (Planck Collaboration et al. 2015).

We fit a spectrum extracted from this region between 0.5-7.0 keV in XSPEC (model wabs* ${ }^{*} f l u x^{*}$ mekal) with the abundance fixed at $Z=0.3 Z_{\odot}$ (Mernier et al. 2017). We found a global temperature of $k T=6.05 \pm 1.13 \mathrm{keV}$ and an X-ray luminosity within $R_{500}$ of $L_{\mathrm{X} 500}=(4.51 \pm 0.33) \times$ $10^{44} \mathrm{erg} \mathrm{s}^{-1}$ in the $0.5-7.0 \mathrm{keV}$ band and $L_{\mathrm{X} 500}=(2.36 \pm$ $0.17) \times 10^{44} \mathrm{erg} \mathrm{s}^{-1}$ in $0.5-2.4 \mathrm{keV}$ band. In the $0.1-2.4 \mathrm{keV}$ band (the ROSAT X-ray band), we found a X-ray luminosity within $R_{500}$ of $L_{\mathrm{X} 500}=(2.77 \pm 0.18) \times 10^{44} \mathrm{erg} \mathrm{s}^{-1}$.

\subsection{XMM-Newton Data}

A959 was observed by the XMM-Newton X-ray Observatory on 12-04-2007 for $41.5 \mathrm{ks}$ (Obs. ID 0406630201). The data were obtained from the XMM-Newton archive and were processed with the epchain and emchain tasks in XMMSAS ${ }^{6} R_{500}=\left(\frac{M_{500}}{500 \rho_{c}(z) 4 \pi / 3}\right)^{1 / 3}$, with $\rho(z)=\frac{E(z)^{2} 3 H_{0}^{2}}{8 \pi G}$ and $E(z)^{2}=$
$\Omega_{\mathrm{M}}(1+z)^{3}+\Omega_{\Lambda}$ version 16.0.0. Periods of background flaring were identified as times for which the total count rate exceeded 0.35 and 0.4 count $\mathrm{s}^{-1}$ for the MOS and PN detectors, respectively. Unfortunately, $\sim 90 \%$ of the data was affected by a strong flare: after filtering periods of high background, only 9.785 ks for the MOS detectors and $4.999 \mathrm{ks}$ for the PN detector remained.

Exposure-corrected images were made with the evselect and eexpmap tasks from the cleaned event lists between the energies of $0.5-2.5 \mathrm{keV}$, where the signal-to-noise of the soft thermal cluster emission is greatest. These images were then used to constrain the emissivity of the dark clump to the south of the main cluster (see Section 3.7). The background in the region of the dark clump is dominated by the cluster emission. For this region, we use as the background count rate the mean count rate in an annulus, centered on the cluster, with inner and outer radii that match those of the dark clump (see Figure 3).

We also extracted a spectrum within the $R_{500}$ region $\left(R_{500}=1099 \mathrm{kpc}\right)$ using the MOS1 data and a local background region that is free of any cluster emission. We fitted the above spectrum in XSPEC with a fixed $N_{\mathrm{H}}$, fixed redshift and fixed abundance $Z=0.3 Z_{\odot}$, and found a temperature of $k T=8.55 \pm 2.30 \mathrm{keV}$ and a X-ray luminosity within $R_{500}$ region of $L_{\mathrm{X} 500}=(4.97 \pm 0.35) \times 10^{44} \mathrm{erg} \mathrm{s}^{-1}$ in the $0.5-7.0 \mathrm{keV}$ band and $L_{\mathrm{X} 500}=(3.24 \pm 0.46) \times 10^{44} \mathrm{erg} \mathrm{s}^{-1}$ in the 0.1-2.4 keV band. Therefore, the Chandra and XMMNewton values for luminosity and temperature agree within the 1- $\sigma$ errors. For convenience, we will use the luminosity derived from the XMM-Newton data in further calculations.

\section{RESULTS AND DISCUSSION}

\subsection{X-ray Properties}

The appearance of the ICM of A959 is fairly smooth, with no cuspy core or other bright substructures (excluding the X-ray point sources, see Figure 3). A number of faint, associated galaxy groups (or subclusters) have been identified previously in ROSAT observations (see, Dahle et al. 2003; Boschin et al. 2009).

The spectral analysis of the X-ray data (see Sections 2.3 and 2.4) indicates that the temperature of the ICM is $k T \approx 6-7 \mathrm{keV}$. There is no evidence of cooler gas in the core. The central density is $n_{e} \approx 2 \times 10^{-3} \mathrm{~cm}^{-2}$ and the central cooling time is $t_{\text {cool }} \approx 3 \times 10^{10}$ yr. A959 is therefore a typical massive NCF cluster. It shows no evidence for possessing a cool corona associated with the BCG, as seen in some NCFs such as the Coma cluster (Sun 2009).

The X-ray luminosity within the $R_{500}$ region in the 0.1-2.4 keV band derived using Chandra and XMM-Newton data (see Section 2.3 Section 2.4) is a factor of three less than the MCXC value from Piffaretti et al. (2011) of $L_{\mathrm{X} 500}=$ $8.37 \times 10^{44} \mathrm{erg} \mathrm{s}^{-1}$ (after correcting for the revised redshift). This factor of three is too large to be due only to the difference in $R_{500}$ used in MCXC catalog $\left(R_{500}=1260 \mathrm{kpc}\right.$ after correcting for redshift, Piffaretti et al. 2011). Instead, the difference might be a result of uncertainties in the modeling that was used for the ROSAT data to correct from aperture flux to $L_{\mathrm{X} 500}$. In support of this possibility, we find a bolometric luminosity within $R_{500}$ from the XMM-Newton 

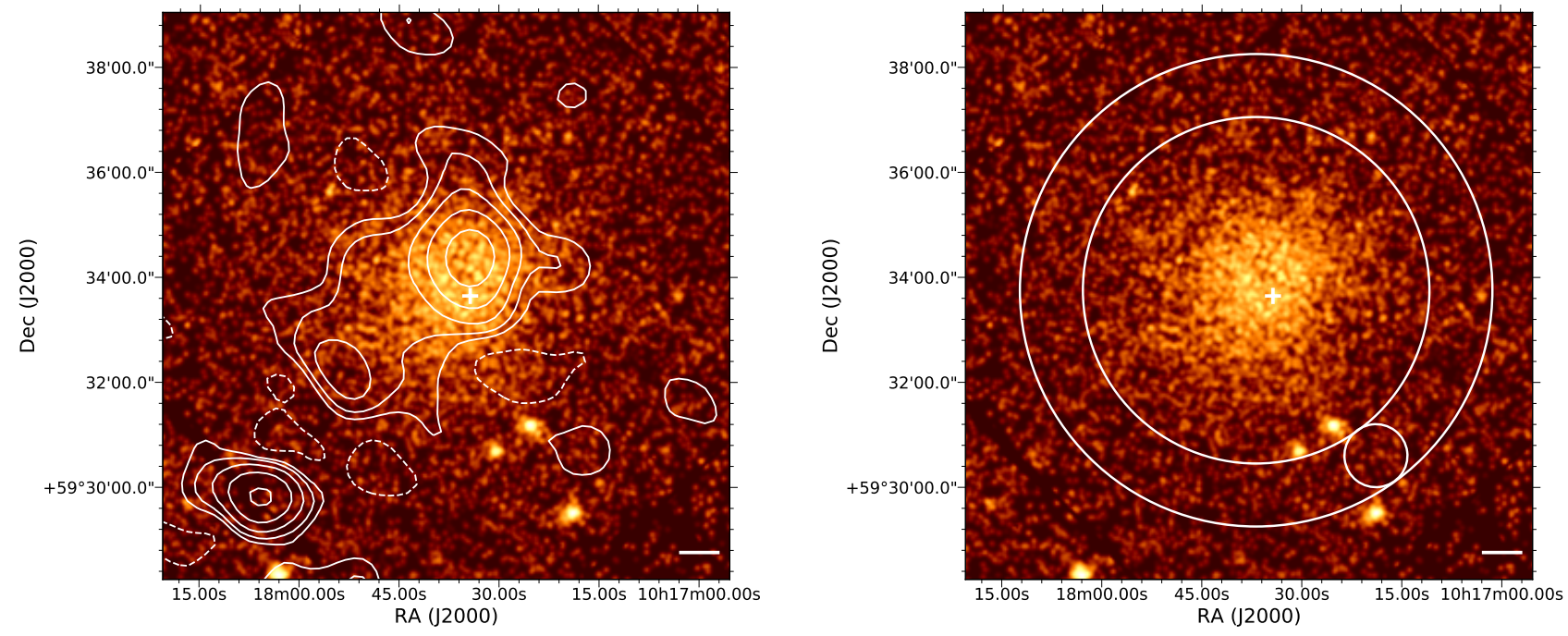

Figure 3. Combined MOS+PN XMM-Newton X-ray image, with the contours from the low-resolution residual LOFAR image (left) and the regions used in the dark-clump analysis (right) overlaid. The image has been smoothed by a Gaussian with FWHM $=5$ pixels The scale bars and symbols are the same as in Figure 1

data of $L_{\mathrm{X}, \mathrm{bol}, 500}=7.1 \times 10^{44} \mathrm{erg} \mathrm{s}^{-1}$, similar to the value derived by Mahdavi et al. (2013), using the same XMMNewton data, of $L_{\mathrm{X}, \text { bol, } 500}=5.8 \times 10^{44} \mathrm{erg} \mathrm{s}^{-1}$, a difference of only $\approx 20 \%$ (see also Connor et al. 2014).

\subsection{The Central Radio Source}

The full-resolution LOFAR image, shown in Figure 1, reveals that the $\mathrm{BCG}$ in the cluster core is a radio galaxy with a bright core (centered on the BCG) and lobes that extend $\sim 100 \mathrm{kpc}$ to the north and south. The total flux density at $143.7 \mathrm{MHz}$, measured from the high-resolution LOFAR image, is $S_{143.7 \mathrm{MHz}}=22.9 \pm 2.3 \mathrm{mJy}$, corresponding to a luminosity of $P_{143.7 \mathrm{MHz}}=(5.8 \pm 0.6) \times 10^{24} \mathrm{~W} \mathrm{~Hz}^{-1}$. The source is also detected in the $322.7 \mathrm{MHz}$ GMRT image, with a flux density of $S_{322.7 \mathrm{MHz}}=9.1 \pm 1.1 \mathrm{mJy}$, implying a spectral index $\left(\alpha\right.$, where $\left.S_{\nu} \propto \nu^{-\alpha}\right)$ between 143.7 and 322.7 $\mathrm{MHz}$ of $\alpha_{143}^{322}=1.14 \pm 0.25$.

Adopting a power-law spectrum with this spectral index, we find a luminosity for the central radio source at 1.4 $\mathrm{GHz}$ of $P_{1400 \mathrm{MHz}}=(4.3 \pm 0.5) \times 10^{23} \mathrm{~W} \mathrm{~Hz}^{-1}$. This luminosity is above the value of the threshold between NCF and CF clusters seen in the B55 and HIFLUGCS cluster samples (Bîrzan et al. 2012).

The interaction between the lobes of the central radio source and the ICM should create X-ray cavities which will rise buoyantly into the ICM. As they are inflated and evolve, they do work on the surrounding ICM. This work is one component of AGN feedback, the maintenance or radio-mode AGN feedback (for reviews see McNamara \& Nulsen 2007; Fabian 2012). Such feedback is rare in NCF systems, although evidence for cavities in a NCF system was recently found in the SPT sample (e.g., SPT-CL J2031-4037, Bîrzan et al. 2017). Therefore, the prevalence and importance of AGN feedback in NCF systems is not well established, but there might often be radio activity and AGN feedback at a low level in such systems. Deeper Chandra data are required to identify any cavities in the ICM of A959.

\subsection{The Giant Radio Halo}

There is clear evidence for diffuse emission to the east of the X-ray core in the low-resolution LOFAR image, shown in Figure 1. This emission extends from the central BCG to the relic, with a largest linear size of $\sim 5$ arcmin=1.3 Mpc, although it does not uniformly fill this region. The total flux density of the halo, excluding the candidate relic (see Section 3.6) and the compact emission from the BCG and the head-tail source to the north of the BCG, is $S_{143 \mathrm{MHz}}=94 \pm 14 \mathrm{mJy}$, corresponding to a luminosity of $P_{143 \mathrm{MHz}}=(2.08 \pm 0.32) \times 10^{25} \mathrm{~W} \mathrm{~Hz}^{-1}$. Using the 1400 $\mathrm{MHz}$ flux density of the diffuse emission measured by Owen et al. (1999) of $S_{1400 \mathrm{MHz}}=3 \times 10^{-3} \mathrm{Jy}$, we find a luminosity of $P_{1.4 \mathrm{GHz}}=0.68 \times 10^{24} \mathrm{~W} \mathrm{~Hz}^{-1}$ and a spectral index of $\alpha_{143}^{1400}=1.48_{-0.23}^{+0.06}$, where the error includes an estimate for the error in the subtraction, adopted to be $50 \%$ of the subtracted flux. ${ }^{7}$ The halo in A959 has a somewhat steeper spectrum than that of the average giant radio halo $(\langle\alpha\rangle \approx 1.3$, Cassano et al. 2013), but we note that our value of $\alpha_{143}^{1400}$ should be treated with caution, as we do not know exactly how the $1400 \mathrm{MHz}$ image of Owen et al. (1999) differs in sensitivity to diffuse emission from our $143 \mathrm{MHz}$ image (e.g., due to different sampling of the uv plane). Also, we do not know if any embedded discrete sources in the $1.4 \mathrm{GHz}$ image were completely subtracted or whether the regions used for the flux-density measurement are identical.

Diffuse radio emission in the form of a giant $\mathrm{RH}$ is often interpreted as evidence of recent, energetic merging activity (Cassano et al. 2013). Such activity is expected in higherredshift systems of X-ray flux limited samples (e.g.; GRHS, EGRHS, Venturi et al. 2007; Kale et al. 2015), such as the one to which A959 belongs (see NORAS, Böhringer et al.

\footnotetext{
7 This spectral index is consistent with the lack of a detection in the residual $322.7 \mathrm{MHz}$ GMRT image, given the noise in this image and the expected flux density of the halo at $322.7 \mathrm{MHz}$.
} 
2000). Below, we compare A959 with other systems which posses giant radio haloes.

\subsection{Scaling Relations for Radio Haloes}

To date, there are approximately 80 systems with detected radio haloes (Feretti et al. 2012; van Weeren et al. 2019). For these systems, the radio luminosity of the $\mathrm{RH}$ is known to scale with a number of cluster properties, the most commonly used of which are the cluster mass, the cluster SZ signal $\left(Y_{\mathrm{SZ}}\right)$, and the X-ray luminosity (see Cassano et al. 2013; Martinez Aviles et al. 2016). ${ }^{8}$ These relations were derived using a sample of $\approx 25$ systems in Cassano et al. (2013) and 41 systems in Martinez Aviles et al. (2016) drawn from the literature, 11 of which are from the GRHS/EGRHS sample (see Venturi et al. 2007, 2008, 2013; Kale et al. 2013, 2015). These RH samples are comprised of systems with a wide range of redshift, mostly between $0.05<z<0.55$, with the notable exceptions of Coma at $z=0.023$ and El Gordo at $z=0.87$.

Additionally, there are a number of RHs known from other studies of single systems and smaller samples that are not present in the above samples (e.g., A399, A401, A2218, A2061, A2065, A2069, PLCKG287.0+32.9, MACS J0416.12403 etc; Feretti et al. 2012; Giovannini \& Feretti 2000; Rudnick \& Lemmerman 2009; Farnsworth et al. 2013; Bonafede et al. 2014a; Ogrean et al. 2015). Also, some systems from the GRHS or EGRHS are not present in the above samples (e.g.; A1682, A2261, RXCJ1314.4-2515, ZwCL5247). Lastly, in the last two years, there has been a rapid increase in studies of individual or small samples of RH systems (see Bernardi et al. 2016; Knowles et al. 2016; Girardi et al. 2016; Venturi et al. 2017; Parekh et al. 2017; Duchesne et al. 2017; Hoang et al. 2017; Wilber et al. 2018; Hlavacek-Larrondo et al. 2018; Cuciti et al. 2018; Savini et al. 2019). We have collected measurements from these studies to form a larger sample of RHs. The systems that are not present in Cassano et al. (2013) or Martinez Aviles et al. (2016) samples are listed in Table 1, with nine of these also present in the Yuan et al. (2015) sample (A399, A2061, A2069, A2218, A3562, ZwCL5247, CL0217+70, H1821+643, and the "Toothbrush" cluster). Table 2 lists the X-ray luminosities; for systems in the Cassano et al. (2013) and Martinez Aviles et al. (2016) samples the cluster masses and the $\mathrm{RH}$ powers are listed in the above papers.

We note that the halo powers in this larger sample have not been derived in a homogeneous way. For example, in some cases the contribution of compact radio sources could not be fully isolated from the RH emission (e.g., A2065 and A2069; Farnsworth et al. 2013) ${ }^{9}$. Also, the cluster Xray luminosities were not derived in a homogenous way: we used the values from Cassano et al. (2013) where available,

8 X-ray luminosity, cluster mass and SZ signal are calculated within $R_{500}$, and the X-ray luminosity is measured in the 0.1 $2.4 \mathrm{keV}$ band.

9 We did not include A2390 from Sommer et al. (2017), as it was not confirmed by LOFAR observations Savini et al. (2019), and A1914 and A2146 since they have only putative RH emission in recent LOFAR observations (Mandal et al. 2019; Hoang et al. 2019b). otherwise we used other samples with derived X-ray luminosities (e.g.; O'Hara et al. 2006; Mantz et al. 2010; Giles et al. 2017; Yuan et al. 2015), or individual papers in some cases when available (e.g.; A1132, ACT-CL J0256.5+0006, CIZA J2242.8+5301; Wilber et al. 2018; Knowles et al. 2016; Hoang et al. 2017) ${ }^{10}$. Otherwise, we used the values from Piffaretti et al. (2011) and even bolometric X-ray luminosity in some cases (e.g.; CL1446+26) ${ }^{11}$. Due to these inhomogeneities, we do not attempt to derive new scaling relations; rather, our goal here is to collect a sample of RHs in order to search for more general trends.

In Figure 4, we plot the halo radio power versus the cluster X-ray luminosity between $0.1-2.4 \mathrm{keV}$ within $\mathrm{R}_{500}$ (see Table 1) and cluster mass within $\mathrm{R}_{500}$ derived from SZ observations (see Table 2) for the larger sample of 80 systems described above (A959 plus the literature systems). However, some systems in this sample do not have X-ray luminosities available in the literature (e.g., PSZ1G018.75+23.57), and hence they do not appear in the right panel of Figure 4. Additionally, others do not have SZ-derived masses available in the literature (e.g., A523, A800, A851, MACS J0416.1-2403, CIZA J2243.8+5301, CL0217+70, CL1446+26) and do thus not appear in the left panel of Figure 4.

Figure 4 shows that there is a large scatter about the above scaling relations (for a discussion see Brunetti et al. 2009; Basu 2012; Cassano et al. 2013; Cuciti et al. 2018). Some of the scatter in the radio power versus X-ray luminosity plot is likely intrinsic, due to for example different systems being caught in different stages of the merger. Significant changes in the X-ray luminosity are expected to occur during and after the merger event (e.g.; Ricker \& Sarazin 2001; Ritchie \& Thomas 2002; Randall et al. 2002; Donnert et al. 2013). In addition, the radio properties of $\mathrm{RHs}$ are predicted to depend on the details of the merger (e.g., mass ratio and energetics) and will evolve during the merger, thus introducing additional scatter (see Cassano et al. 2013; Martinez Aviles et al. 2016; Cuciti et al. 2018).

\subsection{The Relation of Cluster Properties to the Merger State}

To investigate the origins of the scatter seen in Figure 4 further, we can search for relations between the properties of the $\mathrm{RH}$ and the degree to which the X-ray luminosity has been boosted (or suppressed). To this end, we calculate the ratio between the measured X-ray luminosity, $L_{\mathrm{X}}(R<500)$, and the X-ray luminosity predicted from the SZ derived mass, $L_{\text {Xpred }}(R<500)$. To calculate the latter, we use the well-known scaling between the cluster luminosity and cluster mass. There is a large literature on the cluster luminosity-mass $(L M)$ scaling relation and its form (e.g.; Reiprich \& Böhringer 2002; Allen et al. 2003; Pratt et al.

10 For PLZ1G139.61+24.20, PLZ1G108.18-11.53 and ZwCL2341.1+0000 we derived the X-ray luminosity using the archived Chandra data (Obs IDs=15139, 17312, 17490).

11 Some systems are present in more than one of the above studies (O'Hara et al. 2006; Piffaretti et al. 2011; Cassano et al. 2013; Mantz et al. 2010; Giles et al. 2017). In general, the X-ray luminosities between studies are consistent, with a few exceptions where there is a factor of 2 or more difference between studies e.g., A2142, A2261, A141, and A1689. 
2009; Vikhlinin et al. 2009; Mantz et al. 2010; Giles et al. 2017 ), with the slope of the relation varying across studies from 1.3 (Allen et al. 2003; Mantz et al. 2010) to $~ 1.6$ and above (Vikhlinin et al. 2009; Pratt et al. 2009; Mantz et al. 2016; Giles et al. 2017). We use two recent determinations to calculate $L_{\mathrm{Xpred}}$ : the relation of Mantz et al. (2010), which has a $L M$ normalization of $0.82 \pm 0.11$ and a $L M$ slope of $1.29 \pm 0.07$ (see Table 7 of Mantz et al. 2010), and the relation of Giles et al. (2017), which has a slope of $1.92 \pm 0.24$ (see Table 4 and Table B1 of Giles et al. 2017). Both of these relations include corrections for sample biases that account for the tendancy of X-ray selected samples to preferentially include clusters that have higher luminosities than typical for a given mass (for a discussion, see Giles et al. 2017). We plot the halo radio power versus the ratio between the measured X-ray luminosity and that calculated using these scaling relations in Figure 5.

We find that the measured X-ray luminosity is higher on average by a factor of $\sim 1.5-2$ than that predicted by the $L M$ relations, implying that clusters with $\mathrm{RHs}$ tend to be overluminous for a given mass relative to the average over all clusters. One explanation for this overluminosity is that clusters with RHs are preferentially caught in a state soon after a major merger has occurred, when the X-ray luminosity is expected to be boosted (e.g., Donnert et al. 2013). An alternative explanation is that our sample is biased towards overluminous systems, for example due to selection effects. Our sample is largely based on X-ray selected samples, so a sample bias of this kind is possible. Samples of RH systems selected on other properties, such as the cluster mass, would be very useful in understanding whether the overluminosity we observe is an intrinsic property of RH systems or not (Cuciti et al. 2015; Kale 2018).

We note that the values of $L_{\text {Xpred }}(R<500)$ calculated using the scaling relation of Mantz et al. (2010) are $\sim 1.5$ times higher than those calculated using that of Giles et al. (2017) for our sample. This difference is mainly due to the differing slopes between the two relations and the fact that our sample is comprised mostly of clusters with masses below $\sim 10^{15} \mathrm{M}_{\odot}$, where this difference in slope has the greatest effect. There is also an additional smaller systematic offset of $\approx 1.1$ between the luminosities used in Mantz et al. (2010) and Giles et al. (2017) that we do not correct for (for details see Giles et al. 2017). One consequence of this difference is that, for the Mantz et al. (2010) scaling relation, the ratio $L_{\mathrm{X}} / L_{\mathrm{X} \text { pred }}$ falls below unity for a number of systems. Such ratios are not expected in simulations until late in the merging process (e.g., Donnert et al. 2013), well after the $\mathrm{RH}$ should have faded away. Therefore, the low ratios could be interpreted as indirect support for the higher slope of the $L M$ relation of Giles et al. (2017) (which does not result in such low ratios). However, the low ratios could also occur as a consequence of the intrinsic scatter about the $L M$ relation.

To investigate how the measured-to-predicted luminosity ratio relates to the spectral properties of the radio halo, we separated the full sample into two categories: systems with steeper spectral indices $(\alpha>1.5)^{12}$ and systems with

12 The values of $\alpha$ are listed in Table 1 and otherwise were taken from Cassano et al. (2013), plus A2256 (1.6, Brentjens 2008), flatter spectral indices $(\alpha<1.5)^{13}$. These two subsamples are indicated by the different colors in Figure 5 .

We find that $P_{1.4 \mathrm{GHz}}$ appears to be correlated with $L_{\mathrm{X}} / L_{\mathrm{Xpred}}$ in the flatter-halo subsample when using the Mantz et al. (2010) scaling relation. To quantify the strength of this trend, we calculated the Spearman's rank correlation coefficient. We find that the correlation is significant, with a correlation coefficient of 0.70 and a probability that the two quantities are unrelated of $1 \times 10^{-4}$. However, there is no such correlation when the relation of Giles et al. (2017) is used. If present, such a correlation would imply that systems with powerful radio haloes are those for which the X-ray luminosity is most affected (relative to the mass).

However, a difference between the two subsamples is evident in both panels of Figure 5: flatter systems tend to have lower ratios of measured-to-predicted luminosity and higher radio powers than steeper systems (albeit with considerable overlap). It has been proposed that a category of the steepspectrum halos may be formed in low-turbulence mergers (Cassano et al. 2006). Since the radio power of the halo decreases as the merger evolves, at a given radio power steep systems will tend to be observed at an earlier stage of the merger than flatter ones. This expectation is consistent with the observed distribution of steep halos in Figure 5, as systems observed at an earlier stage are also expected to have a higher ratio of measured-to-predicted X-ray luminosity than those observed at later stages, when the X-ray luminosity has decreased. Therefore, the tendency for steep-spectrum, low-power halo systems to have high ratios of measured-topredicted X-ray luminosity is broadly consistent with this scenario.

In support of this interpretation, the steep systems with the lowest $\mathrm{RH}$ power in our sample are A3562 and A2811, which are also the lowest-mass systems in the sample. As a result, mergers in these systems are expected to be less turbulent than in high-mass systems (Cassano et al. 2006). Other low-power, steep-spectrum RHs in the plot are the recently identified RHs A1132, RXC J0142.0+2131, RXJ1720.1+2638 and PSZ1G139.61+24.20 (Wilber et al. 2018; Savini et al. 2019), located in the lower-right corner. These RHs were interpreted as likely having been created in lower-turbulence merger events. They all have high ratios of measured-to-predicted X-ray luminosity, suggesting they were caught in a stage that is fairly close to the core passage.

Therefore, the combination of the ratio of measuredto-predicted X-ray luminosity and the spectral properties of the halo appears to be a general indicator of the merger stage. Further support for this interpretation comes from the location of halo systems with radio relics in the plot.

A2255 (1.6, Pizzo \& de Bruyn 2009), RXC J1514.9-1523 (1.6, Giacintucci et al. 2011), and A2034 (1.7, Shimwell et al. 2016).

13 The values of $\alpha$ are listed in Table 1 and otherwise are as follows: A2744 (1.43, Pearce et al. 2017), A2163 (1.18, Feretti et al. 2004), RXC J2003.5-2223 (1.3, Giacintucci et al. 2009), A520 (1.12, Vacca et al. 2014; Hoang et al. 2019a), A1758N (1.2, Botteon et al. 2018), A2219 (0.9, Orrú et al. 2007), A665 (1.04, Feretti et al. 2004), Coma (1.34, Kim et al. 1990), the Bullet cluster (1.5, Shimwell et al. 2014), MACS J0717.5+3745 (1.4, Bonafede et al. 2018), El Gordo (1.2, Lindner et al. 2014), MACS J1752.5+4440 (1.33, Bonafede et al. 2012), and A3888 (1.48, Shakouri et al. 2016). 
We indicate such systems in Figure 5 (triangles): it is clear that systems with relics tend to have low ratios of measuredto-predicted X-ray luminosity at a given halo radio power, especially relative to other systems of the same spectral class (i.e., flat or steep). This tendency is in line with merger simulations (e.g., Vazza et al. 2012; Ha et al. 2018) that posit that relics are generally found at a late stage of the merger ( 1 Gyr after core passage), when the shock has propagated to large enough radii $(\sim 1 \mathrm{Mpc})$ that the Mach number is sufficiently high to efficiently create the relics. At these later stages, the X-ray luminosity and halo radio power have decreased, and the relic systems therefore tend to lie to the left of younger (non-relic) systems in Figure 5.

Lastly, in Figure 5 (right panel), we plot the RH upper limits from Cassano et al. (2013) and Kale et al. (2015). There are 20 such systems in Cassano et al. (2013) and two more systems in Kale et al. (2015) which have masses derived from SZ observations. However, in 4 out of the 20 upper limits systems from Cassano et al. (2013) have been detected RH emission (e.g., A141, A2146, A2261, and RXCJ0142.0+2131, see Table 1 for references), and as a result, they do not belong to the upper limits class category. Furthermore, we did not include in the RH upper-limit sample the strong cooling flow systems without signs of merging activity (e.g., AS780, A3088, RXCJ1115.8+0129). As a result, we have a sample for the RH upper limits of 13 systems (see Table 2). Figure 5 shows that systems with upper limits share the same region of the plot as the steep-spectrum RHs, in line with steep-spectrum RH formation models (e.g.; Brunetti et al. 2009; Cassano et al. 2010a) that posit that some of these systems may have faint, steep-spectrum haloes that remain undetected in current observations.

\subsection{The Candidate Radio Relic}

One of the most prominent diffuse features in both the lowand high-resolution LOFAR images of A959 is the linear feature, $\sim 400 \mathrm{kpc}$ in length and $\sim 125 \mathrm{kpc}$ in width, located $\sim 800 \mathrm{kpc}$ to the south-east of the cluster core. The location, orientation, and elongated, linear morphology of this feature strongly resembles those of cluster radio relics. In support of this scenario, there are no obvious optical counterparts that could explain the emission as being associated with a radio galaxy.

As discussed in the introduction, radio relics are thought to be created in merging systems, when electrons are accelerated or re-accelerated by the merger shocks. There are a number of halo systems that show evidence of $\mathrm{X}$-ray shocks associated with the radio relic emission (e.g. A521, Bullet, A754, El Gordo, A2146; Giacintucci et al. 2006, 2008; Shimwell et al. 2015; Macario et al. 2011; Botteon et al. 2016; Russell et al. 2010; Hlavacek-Larrondo et al. 2018). Such shocks, thought to be generated during the merger, are typically found to lie roughly perpendicular to the merger axis, often at the outskirts of the cluster. The complex distribution of mass and galaxies in A959 makes it difficult to determine the merger axis, but there is an elongation in the weak-lensing maps in the direction of the relic that could indicate that the merger axis is along this line (Dahle et al. 2003; Boschin et al. 2009). In this case, the putative relic meets many of the characteristics of known relics: it lies $\sim 1 \mathrm{Mpc}$ from the cluster center, and thus at the cluster outskirts; it is located roughly along the merger axis; and its long axis is oriented perpendicular to the merger axis. However, confirmation that it is a relic requires radio data at higher frequencies to confirm that the spectral and polarization properties are that of a relic.

We measure the luminosity of the putative relic to be $P_{142 \mathrm{MHz}}=(2.85 \pm 0.32) \times 10^{24} \mathrm{~W} \mathrm{~Hz}^{-1}$. We do not detect the relic in a lower-resolution $322.7 \mathrm{MHz}$ GMRT image (with a restoring beam of $39^{\prime \prime} \times 50^{\prime \prime}$ ), implying a lower limit on the spectral index of $\alpha>0.7$. There is no evidence in either the Chandra or XMM-Newton images of a surface-brightness edge in the region of the relic that would be indicative of a shock associated with it. However, both exposures have few counts $\left(\lesssim 0.2\right.$ counts pixel ${ }^{-1}$, where 1 pixel $=0.4919$ arcsec on a side for Chandra and 1.1 arcsec on a side for XMM-Newton) at this location, and any edge produced by a typical shock would not be visible. Therefore, deeper Xray data are needed to confirm the presence of a shock at this location.

\subsection{The Dark Clump}

Dahle et al. (2003) identified a possible dark mass clump in their weak-lensing map of A959. The clump, designated WL $1017.3+5931$, lies to the south-west of the cluster center and has little-to-no associated X-ray emission or galaxy overdensity (Boschin et al. 2009). We can place limits on the X-ray gas mass fraction in the clump using the XMM-Newton data (we do not use the Chandra data for this purpose as they are shallower and therefore any limits derived from them would be less constraining).

To this end, we measured the count rates in the exposure-corrected XMM-Newton images discussed in Section 2.4 in the dark-clump and background regions shown in Figure 3. The dark-clump region was chosen to encompass the majority of the mass peak found by Dahle et al. (2003) while excluding the nearby X-ray point source and has a radius of $r=156 \mathrm{kpc}$ at the redshift of A959. For the background emission in the region of the dark clump, which is comprised of the local background emission from the main cluster and the instrumental background, we used the mean count rate in an annulus centered on the cluster with inner and outer radii that match those of the dark-clump region (see Figure 3).

In the dark-clump region, we measure an upper limit on the background-subtracted count rate, summed over all three detectors, of $(1.6 \pm 5.4) \times 10^{-6}$ count $\mathrm{s}^{-1}$ pixel $^{-1}$. Therefore, we do not detect significant excess emission from the dark clump. To place limits on the density of X-ray gas in the clump, we obtained predicted count rates from PIMMS (the Portable, Interactive Multi-Mission Simulator ${ }^{14}$ ), using the APEC thermal plasma model with a temperature of $3 \mathrm{keV}$ and an abundance of 0.3 times the solar abundance. We adjusted the normalization of the APEC model to match the upper limit on the count rate in the dark-clump region, accounting for the encircled-energy fraction of the
14 See https://heasarc.gsfc.nasa.gov/cgi-bin/Tools/ w3pimms/w3pimms.pl 

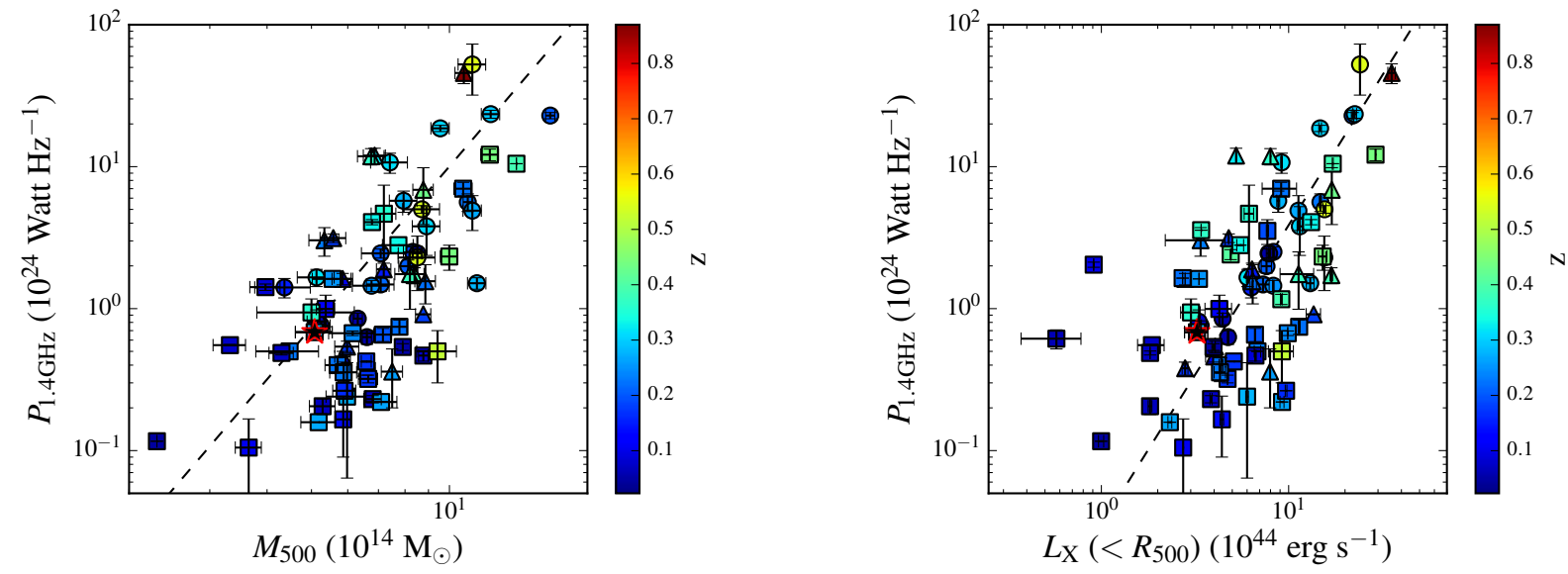

Figure 4. The monochromatic $1.4 \mathrm{GHz}$ radio power versus the SZ-derived cluster mass $M_{500}$ (left panel) and versus the X-ray luminosity in the 0.1-2.4 keV band $L_{\mathrm{X}}\left(<R_{500}\right)$ (right panel), both derived within $R_{500}$. Except for A959 (denoted by the red star), the values for the systems are taken from the literature (see Table 1 and Table 2). However, for El Gordo we used the radio halo power from Lindner et al. (2014). Circles denote the systems from the Cassano et al. (2013) sample, triangles denote the extra 16 systems from Martinez Aviles et al. (2016) sample and the squares denote the systems from Table 1. The dashed lines show the best-fit relations of Cassano et al. (2013). Some systems do not appear in the left panel since there are no available SZ-derived masses, while others do not appear in the right panel since there are no published X-ray luminosities (e.g; PSZ1G018.75+23.57).
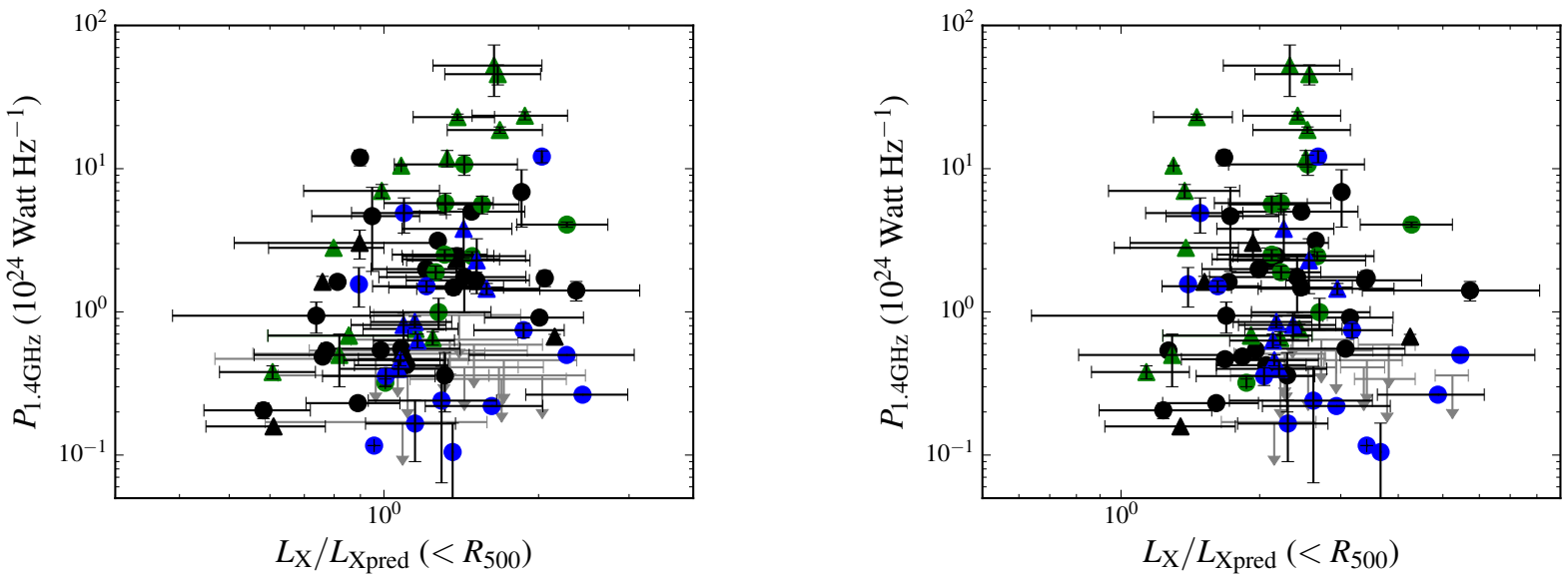

Figure 5. The monochromatic $1.4 \mathrm{GHz}$ radio power versus the ratio between the measured X-ray luminosity and the predicted X-ray luminosity from the SZ-derived cluster mass using the LM scaling relations of Mantz et al. (2010) (left panel) and Giles et al. (2017) (right panel). The green symbols denote the systems with flatter-spectrum $(\alpha<1.5)$ haloes, the blue symbols denote the steeper-spectrum $(\alpha>1.5)$ haloes, and the black symbols denote the systems that lack spectral information in the literature. The triangle symbols denote the RHs plus relic (radio shock) systems, and the circle symbols denote the systems that do not have relic emission. In grey we overplot the upper limits from the merging systems without detected RH emission (Cassano et al. 2013; Kale et al. 2015).

point spread function for this region $(\approx 0.8) .{ }^{15}$ The upper limit is defined as three times the uncertainty of the background count rate in the region (i.e., the 3- $\sigma$ upper limit, $1.6 \times 10^{-5}$ count s$^{-1}$ pixel $^{-1}$ ).

From the resulting normalization, and assuming the gas fills a sphere with uniform density, we find the limit on the offaxisxraypsf.html. electron density in the dark clump of $n_{\mathrm{e}}<3 \times 10^{-4} \mathrm{~cm}^{-2}$. This density implies a total gas mass of $M_{\text {gas }}<1.2 \times$ $10^{11} M_{\odot}$ (assuming $n=2 n_{\mathrm{e}}$ ). Dahle et al. (2003) report a total mass for the dark clump of $M_{\text {tot }}=1.2 \times 10^{14} M_{\odot}$ within a radius of $r=230 \mathrm{kpc}$ (adjusted to our adopted cosmological parameters). Again assuming spherical geometry and a uniform density to adjust for the slightly different radii $(r=230 \mathrm{kpc}$ for the total mass and $r=156 \mathrm{kpc}$ for the gas mass), we find the upper limit on the gas mass fraction 
within $r=156 \mathrm{kpc}$ to be $f_{\text {gas }}<1.7 \times 10^{-3}$. However, the total mass estimate should be treated with caution since only weak lensing data were used (see A2744, Jauzac et al. 2016). Nevertheless, the low gas mass fraction implies the clump, if real, was efficiently stripped of its X-ray gas, similar to other, X-ray gas-poor mass concentrations (e.g., Jee et al. 2014; Wang et al. 2016; Jee et al. 2016).

In addition to A959, there are a number of other systems in which a dark clump has been reported, e.g., A2744 and A520 (see also the review of Wittman et al. 2018). However, for A2744 the dark clump reported in Merten et al. (2011) was not confirmed by Jauzac et al. (2016), who used both weak- and strong-lensing data. For A520 the results are also controversial, with some works detecting a dark clump (Mahdavi et al. 2007; Okabe \& Umetsu 2008; Jee et al. 2012, 2014) and others finding no significant detection (Clowe et al. 2012; Peel et al. 2017).

\section{SUMMARY}

Using LOFAR Two-meter Sky Survey (LoTSS) data we have identified a radio halo and likely radio relic in A959. The $\mathrm{RH}$ has a flux at $144 \mathrm{MHz}$ of $S_{143.7 \mathrm{MHz}}=0.094 \pm 0.014 \mathrm{Jy}$. Using the measured flux at $1400 \mathrm{MHz}$ for all diffuse emission from Owen et al. (1999), we found a spectral index for the RH of $1.48_{-0.23}^{+0.06}$. Additionally, we report the detection of a likely radio relic in $\mathrm{A} 959, \sim 400 \mathrm{kpc}$ in length and $\sim 125 \mathrm{kpc}$ in width, located $\sim 800 \mathrm{kpc}$ to the south-east of the cluster core. There is no indication of a surface brightness edge in the actual Chandra and XMM-Newton data, but both have very few counts at the relic location $\left(\lesssim 0.2\right.$ counts pixel $\left.{ }^{-1}\right)$. Deeper X-ray data will be required to search for shocks in the ICM at the relic location.

We also examined the putative dark clump WL $1017.3+5931$ for which no associated galaxy concentration has been identified (Dahle et al. 2003). Using the XMM Xray data and the total mass from Dahle et al. (2002), we placed limits on the X-ray gas mass fraction in the clump. We find the upper limit on the gas mass fraction within $r=156 \mathrm{kpc}$ to be $f_{\text {gas }}<1.7 \times 10^{-3}$, implying efficient stripping of the gas. However, this value (and the existence of the clump itself) should be treated with caution, since only weak-lensing data were used to measure the mass distribution, which consequently could have significant uncertainties (see, e.g., A2744 and A520, Jauzac et al. 2016; Clowe et al. 2012; Peel et al. 2017).

To place the diffuse radio emission in A959 in context, we collected all known $\mathrm{RH}$ detections from the literature $(80$ systems in total) and added A959 to plots between the nonthermal and thermal power (e.g., Cassano et al. 2013; Martinez Aviles et al. 2016) of this full RH sample. We find that the RH of A959 falls close to the scaling relations of Cassano et al. (2013). As previously reported (Brunetti et al. 2009; Basu 2012; Cassano et al. 2013; Kale et al. 2015; Cuciti et al. 2018), there is a large scatter in these scaling relations. This scatter may be partly explained as being due to evolution in the radio and X-ray luminosities during the merger (e.g., Ricker \& Sarazin 2001; Ritchie \& Thomas 2002; Randall et al. 2002; Donnert et al. 2013).

To investigate such evolution, we examined how the halo radio power relates to the ratio between the measured
X-ray luminosity and that predicted from the SZ cluster mass using the cluster $L M$ scaling relations of Mantz et al. (2010) and Giles et al. (2017), and we summarize the results below:

- We find evidence that the flat-spectrum haloes occur in systems with lower X-ray luminosity ratios and higher halo radio powers, while the steep-spectrum haloes tend to occur in systems with higher $X$-ray luminosity ratios and lower radio powers. We argue that this result is consistent with the expectations of turbulent re-acceleration models of halo formation (e.g., Brunetti et al. 2009; Cassano et al. 2010a), where the halo spectral steepness is strongly influenced by the level of turbulence generated by the merger. Specifically, in these models, steep-spectrum haloes are expected to be created preferentially in low-turbulence mergers (Cassano et al. 2006), where the expected lifetime of the halo is short. The short lifetimes imply that such systems (e.g; RXJ1720.1+2638, Savini et al. 2019) are more likely to be observed at an earlier stage of the merger than the systems with longer-lived, flatter haloes.

- We also find evidence that the RH systems with radio relics have lower measured-to-predicted X-ray luminosities than similar non-relic systems. This finding is consistent with simulations of relics (e.g., Vazza et al. 2012; Ha et al. 2018), which find that relics tend to be observed in the cluster outskirts at the later stages of the merger, when the $\mathrm{X}$-ray luminosity is expected to have decreased significantly.

We therefore posit that the combination of measuredto-predicted X-ray luminosity and the spectral properties of the $\mathrm{RH}$ is a general indicator of the merger stage, in line with simulations (Ritchie \& Thomas 2002; Donnert et al. 2013). 
Table 1. Radio Halo properties for the additional systems

\begin{tabular}{|c|c|c|c|c|c|c|}
\hline System $^{a, b}$ & $z$ & $\begin{array}{c}M_{\mathrm{SZ} 500} \\
\left(10^{14} \mathrm{M}_{\odot}\right)\end{array}$ & $\begin{array}{l}\text { Freq. } \\
(\mathrm{MHz})\end{array}$ & $\begin{array}{l}\text { Flux density } \\
\qquad(\mathrm{mJy})\end{array}$ & $\alpha^{c}$ & $\begin{array}{c}P_{1.4 \mathrm{GHz}^{d}} \\
\left(10^{24} \mathrm{~W} \mathrm{~Hz}^{-1}\right)\end{array}$ \\
\hline A959 & 0.288 & $5.08 \pm 0.47(2)$ & 143.7 & $94 \pm 14(6)$ & $1.48(6)$ & $0.68(31)$ \\
\hline $\mathrm{A} 141^{U}$ & 0.23 & $4.48 \pm 0.7(5)$ & 168 & $110 \pm 11(17)$ & $>2.1(17)$ & $<0.5$ \\
\hline A399 & 0.0718 & $5.29 \pm 0.34(5)$ & 1400 & $16 \pm 2(29)$ & $\ldots$ & $0.21 \pm 0.03$ \\
\hline A401 & 0.0737 & $6.84 \pm 0.32(4)$ & 1400 & $17 \pm 1(8)$ & $\ldots$ & $0.23 \pm 0.01$ \\
\hline A523 & 0.104 & $\ldots$ & 1400 & $72 \pm 3(23)$ & $\ldots$ & $2.04 \pm 0.08$ \\
\hline A800 & 0.2223 & $\ldots$ & 1400 & $10.6(24)$ & $\ldots$ & 1.64 \\
\hline A $851^{*}$ & 0.4069 & $\ldots$ & 1400 & $3.7 \pm 0.3(21)$ & $\ldots$ & $2.41 \pm 0.20$ \\
\hline $\mathrm{A} 1132^{U}$ & 0.1369 & $5.87 \pm 0.22(4)$ & 145 & ... & $1.75(41)$ & $0.17 \pm 0.08$ \\
\hline A1451 & 0.199 & $7.16 \pm 0.32$ & 1500 & $5.0 \pm 0.6(14)$ & $>1.3(14)$ & $<0.66 \pm 0.07$ \\
\hline A1550 & 0.254 & $5.55 \pm 0.54(5)$ & 1400 & $7.7(24)$ & - & 1.62 \\
\hline $\mathrm{A} 1682^{U}$ & 0.226 & $5.70 \pm 0.35$ & 240 & $46 \pm 4(39)$ & $1.7(28)$ & $0.40 \pm 0.05$ \\
\hline A2061 & 0.0777 & $3.32 \pm 0.27(5)$ & 300 & $270 \pm 2(33)$ & $\ldots$ & $0.55 \pm 0.01$ \\
\hline A2065 & 0.073 & $4.30 \pm 0.26(5)$ & 1400 & $32.9 \pm 11(18)$ & $\ldots$ & $0.48 \pm 0.02$ \\
\hline A2069 & 0.116 & $5.45 \pm 0.37(5)$ & 1400 & $28.8 \pm 7.2(18)$ & $0.93(16)$ & $1.00 \pm 0.02$ \\
\hline A2142 & 0.089 & $8.77 \pm 0.21$ & 1400 & $23 \pm 2(40)$ & $\ldots$ & $0.47 \pm 0.04$ \\
\hline A2218 & 0.1756 & $6.59 \pm 0.164(4)$ & 1400 & $4.7(20)$ & 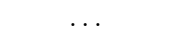 & 0.43 \\
\hline $\mathrm{A} 2261^{U}$ & 0.224 & $7.78 \pm 0.30(4)$ & 1400 & $4.37 \pm 0.35$ & $1.7(34)$ & $0.75 \pm 0.06$ \\
\hline $\mathrm{A} 2811^{U}$ & 0.1079 & $3.65 \pm 0.24(4)$ & 168 & $80.7 \pm 16.5(17)$ & $>1.5(17)$ & $<0.11 \pm 0.06$ \\
\hline $\mathrm{A} 3562^{U}$ & 0.049 & $2.3(3)$ & 1400 & $20(37)$ & $1.56(19,37)$ & 0.12 \\
\hline ACT-CLJ0256.5+0006 & 0.363 & $5.0 \pm 1.2(1)$ & 610 & $5.6 \pm 1.4(27)$ & $\ldots$ & $0.94 \pm 0.23$ \\
\hline AS1121* & 0.358 & $7.19 \pm 0.45(4)$ & 168 & $154 \pm 48(17)$ & $\cdots$ & $4.66 \pm 2.75$ \\
\hline CIZA J0638.1+4747 & 0.174 & $6.65 \pm 0.34$ & 1500 & $3.3 \pm 0.2(14)$ & $>1.3(14)$ & $<0.32 \pm 0.02$ \\
\hline CIZA J2242.8+5301* & 0.192 & $\ldots$ & 145 & $346 \pm 64(25)$ & $1.03(25)$ & $3.1 \pm 1.0$ \\
\hline CL0217+70 & 0.0655 & $\ldots$ & 1400 & $58.6 \pm 0.9(12)$ & $\ldots$ & $0.61 \pm 0.09$ \\
\hline CL1446+26* & 0.370 & $\ldots$ & 1400 & $7.7(24)$ & $\ldots$ & 3.57 \\
\hline $\mathrm{H} 1821+643$ & 0.332 & $6.78 \pm 0.27(4)$ & 1665 & $19.9 \pm 0.5(11)$ & $1.1(11)$ & $4.07 \pm 0.17$ \\
\hline MACS J0416.1-2403 ${ }^{U}$ & 0.393 & $\ldots$ & 1500 & $1.58 \pm 0.13(30)$ & $1.6(30)$ & $1.16 \pm 0.09$ \\
\hline MACS J0417.5-1154 ${ }^{U}$ & 0.443 & $12.25 \pm 0.55(4)$ & 1575 & $10.6 \pm 1.0(32)$ & $1.72(32)$ & $12.15 \pm 1.15$ \\
\hline MACS J2243.3-0935 & 0.44 & $9.99 \pm 0.44(1)$ & 610 & $10.0 \pm 2.0(13,32)$ & $\ldots$ & $2.41 \pm 0.28$ \\
\hline PLCKG004.5-19.5 & 0.516 & $9.42 \pm 0.94(5)$ & 610 & $1.2 \pm 0.5(7)$ & $1.2 \pm 0.4(7)$ & $0.5 \pm 0.2$ \\
\hline PLCKG287.0+32.9 & 0.39 & $14(2)$ & 150 & $314(10)$ & $1.28(10)$ & 10.5 \\
\hline PSZ1G018.75+23.57 & 0.089 & $3.97 \pm 0.30(4)$ & 1860 & $48.3 \pm 2.5(9)$ & $\ldots$ & $1.42 \pm 0.07$ \\
\hline PSZ1G108.18-11.53 & 0.335 & $7.74 \pm 0.60$ & 1380 & $6.8 \pm 0.2(15)$ & $1.4 \pm 0.1$ & $2.8 \pm 0.1$ \\
\hline PSZ1G139.61+24.20U & 0.27 & $7.09 \pm 0.60(5)$ & 144 & $\ldots$ & $>1.7(34)$ & $<0.22(34)$ \\
\hline RXC J0142.0+2131 $U$ & 0.28 & $5.98 \pm 0.60(4)$ & 144 & $32 \pm 6(34)$ & $>1.6(34)$ & $<0.24$ \\
\hline RX J0603.3+4214* & 0.225 & $10.72 \pm 0.49$ & 1500 & $46 \pm 5(36)$ & $1.08(36)$ & $7.00 \pm 0.76$ \\
\hline RXC J1314.4-2515 & 0.228 & $6.15 \pm 0.7(3)$ & 610 & $10.3 \pm 0.3(38)$ & $\ldots$ & $0.67 \pm 0.03$ \\
\hline RXJ1720.1+2638 & 0.164 & $5.90 \pm 0.34(4)$ & 144 & & $>1.5(34)$ & $<0.264(34)$ \\
\hline Triangulum Aus. & 0.051 & $7.94 \pm 0.15$ & 1330 & $92 \pm 5(9)$ & $\ldots$ & $0.54 \pm 0.03$ \\
\hline ZwCL2341.1+0000 & 0.27 & $5.18 \pm 0.44$ & 1400 & $10(22)$ & $\ldots$ & 0.16 \\
\hline $\mathrm{ZwCL5247*U}$ & 0.229 & $5.88 \pm 0.40$ & 1400 & $2.0 \pm 0.3(26)$ & $1.7(26)$ & $0.35 \pm 0.05$ \\
\hline
\end{tabular}

References: SZ References: (1) Hasselfield et al. (2013); (2) Planck Collaboration et al. (2014); (3) Planck Collaboration et al. (2015); (4) Planck Collaboration et al. (2016); (5) SZ-Cluster Database (see http: //szcluster-db.ias.u-psud.fr). Radio References: (6) this work; (7) Albert et al. (2017); (8) Bacchi et al. (2003) (9) Bernardi et al. (2016); (10) Bonafede et al. (2014a); (11) Bonafede et al. (2014b); (12) Brown et al. (2011); (13) Cantwell et al. (2016); (14) Cuciti et al. (2018); (15) de Gasperin et al. (2015); (16) Drabent et al. in press; (17) Duchesne et al. (2017); (18) Farnsworth et al. (2013); (19) Giacintucci et al. (2005); (20) Giovannini \& Feretti (2000); (21) Giovannini et al. (2009); (22) Giovannini et al. (2010); (23) Girardi et al. (2016); (24) Govoni et al. (2012); (25) Hoang et al. (2017); (26) Kale et al. (2015); (27) Knowles et al. (2016); (28) Macario et al. (2013); (29) Murgia et al. (2010); (30) Ogrean et al. (2015); (31) Owen et al. (1999); (32) Parekh et al. (2017); (33) Rudnick \& Lemmerman (2009); (34) Savini et al. (2019); (35) Sommer et al. (2017); (36) van Weeren et al. (2016a); (37) Venturi et al. (2003); (38) Venturi et al. (2007); (39) Venturi et al. (2013); (40) Venturi et al. (2017); (41) Wilber et al. (2018).

${ }^{a}$ The radio halo systems (taken from the literature) that are not present in the Cassano et al. (2013) and Martinez Aviles et al. (2016) samples. The asterisk marks systems with alternative names: A851 (CL0939+47); AS1121 (SPT-CL J2325-4111); CL1446+26 (ZwCL1447+2619); CIZA J2242.8+5301 (the "Sausage" cluster), ZwCL5247 (RXC J1234.2+0947), RXC J0603.3+4214 (the "Toothbrush" cluster). The 'U' marks the systems with steep-spectrum RHs $(\alpha>1.5)$.

${ }^{b}$ However, there are some candidate haloes that are not present here, e.g.; A2680, A2693, AS84, RXC J2351.0-1954, GMBCG J357.91841-08.97978 (Duchesne et al. 2017); A2552, ZwCL1953 (Kale et al. 2015).

${ }^{c}$ Spectral index from the literature.

${ }^{d}$ Radio luminosity at $1.4 \mathrm{GHz}$ using the spectral indices from the literature when available and adopting $\alpha=1.3$ otherwise. 
Table 2. X-ray Luminosity values for the total sample

\begin{tabular}{|c|c|c|c|c|c|}
\hline System $^{a}$ & $z$ & $\begin{array}{c}L_{\mathrm{X} 500}[0.1-2.4 \mathrm{keV}]^{b} \\
\left(10^{44} \mathrm{erg} \mathrm{s}^{-1}\right)\end{array}$ & $\begin{array}{c}L_{\mathrm{X} \text { pred }}^{\mathrm{M}} \\
\left(10^{44} \mathrm{erg} \mathrm{s}^{-1}\right)\end{array}$ & $\begin{array}{c}L_{\mathrm{X} \text { pred }}^{\mathrm{G}}{ }^{d} \\
\left(10^{44} \mathrm{erg} \mathrm{s}^{-1}\right)\end{array}$ & Relics $^{e}$ \\
\hline A959 & 0.288 & $3.24 \pm 0.46(1)$ & $3.80 \pm 1.02$ & $1.69 \pm 0.55$ & (1) \\
\hline $\mathrm{A} 141^{U}$ & 0.23 & $6.82 \pm 0.27(5)$ & $3.01 \pm 1.06$ & $1.25 \pm 0.56$ & .. \\
\hline A399 & 0.0718 & $1.82 \pm 0.042(18)$ & $3.12 \pm 0.73$ & $1.47 \pm 0.40$ & $\ldots$ \\
\hline A401 & 0.0737 & $3.85 \pm 0.05(11)$ & $4.33 \pm 0.90$ & $2.39 \pm 0.56$ & $\cdots$ \\
\hline A523 & 0.104 & $0.91(12)$ & $\ldots$ & $\ldots$ & $\ldots$ \\
\hline A 800 & 0.2223 & $2.72(12)$ & $\ldots$ & $\ldots$ & $\ldots$ \\
\hline A851* & 0.4069 & $4.91(12)$ & $\ldots$ & $\ldots$ & $\ldots$ \\
\hline $\mathrm{A} 1132^{U}$ & 0.1369 & $4.4 \pm 0.1(16)$ & $3.83 \pm 0.76$ & $1.91 \pm 0.42$ & $\ldots$ \\
\hline A1451 & 0.199 & $6.61(12)$ & $5.3 \pm 1.10$ & $2.98 \pm 0.70$ & $(26)$ \\
\hline A 1550 & 0.254 & $3.32(12)$ & $4.09 \pm 1.13$ & $1.94 \pm 0.65$ & $\ldots$ \\
\hline $\mathrm{A} 1682^{U}$ & 0.226 & $4.36 \pm 0.11(6)$ & $4.09 \pm 0.94$ & $1.98 \pm 0.53$ & $(51)$ \\
\hline A2061 & 0.0777 & $1.86 \pm 0.30(18)$ & $1.72 \pm 0.44$ & $0.61 \pm 0.18$ & $\ldots$ \\
\hline A2065 & 0.073 & $1.82(14)$ & $2.40 \pm 0.55$ & $0.99 \pm 0.27$ & $\ldots$ \\
\hline A2069 & 0.116 & $4.27 \pm 0.69(18)$ & $3.34 \pm 0.70$ & $1.58 \pm 0.38$ & $\ldots$ \\
\hline A 2142 & 0.089 & $6.65 \pm 0.05(11)$ & $6.11 \pm 1.10$ & $3.95 \pm 0.77$ & $\ldots$ \\
\hline A 2218 & 0.1756 & $5.1 \pm 0.5(9)$ & $4.62 \pm 0.84$ & $2.47 \pm 0.49$ & $\ldots$ \\
\hline $\mathrm{A} 2261^{U}$ & 0.224 & $11.38 \pm 0.13(6)$ & $6.09 \pm 1.21$ & $3.58 \pm 0.80$ & $\ldots$ \\
\hline $\mathrm{A} 2811^{U}$ & 0.1079 & $2.73(13)$ & $2.01 \pm 0.47$ & $0.75 \pm 0.21$ & $\ldots$ \\
\hline $\mathrm{A} 3562^{U}$ & 0.049 & $0.997 \pm 0.032(11)$ & $1.04 \pm 0.16$ & $0.29 \pm 0.04$ & .. \\
\hline ACT-CLJ0256.5+0006 & 0.363 & $3.01 \pm 0.36(8)$ & $4.08 \pm 1.87$ & $1.78 \pm 1.09$ & $\ldots$ \\
\hline AS1121* & 0.358 & $6.14 \pm 0.37(3)$ & $6.48 \pm 1.49$ & $3.56 \pm 0.96$ & $\ldots$ \\
\hline CIZA J0638.1+4747 & 0.174 & $4.72(12)$ & $4.69 \pm 1.01$ & $2.52 \pm 0.63$ & $\ldots$ \\
\hline CIZA J2242.8+5301* & 0.192 & $7.7 \pm 0.1(7)$ & $\ldots$ & $\ldots$ & $(7,43)$ \\
\hline CL0217+70 & 0.0655 & $0.575 \pm 0.202(18)$ & $\ldots$ & $\ldots$ & $\ldots$ \\
\hline CL1446+26* & 0.370 & $3.42(16)$ & $\ldots$ & $\ldots$ & $\ldots$ \\
\hline $\mathrm{H} 1821+643$ & 0.332 & $13.18 \pm 0.03(18)$ & $5.81 \pm 1.17$ & $3.08 \pm 0.70$ & $\ldots$ \\
\hline MACS J0416.1-2403 ${ }^{U}$ & 0.393 & $9.14 \pm 0.10(10)$ & $\ldots$ & & $\ldots$ \\
\hline MACS J0417.5-1154 & 0.443 & $29.1(12)$ & $14.34 \pm 2.98$ & $10.86 \pm 2.57$ & $\ldots$ \\
\hline MACS J2243.3-0935 & 0.44 & $15.2 \pm 0.8(9)$ & $10.98 \pm 2.27$ & $7.32 \pm 1.72$ & $(24)$ \\
\hline PLCKG004.5-19.5 & 0.516 & $9.2 \pm 1.4(2)$ & $11.23 \pm 3.13$ & $7.13 \pm 2.43$ & $(2)$ \\
\hline PLCKG287.0+32.9 & 0.39 & $17.2 \pm 0.11$ & $15.92 \pm 2.39$ & $13.23 \pm 1.98$ & $(20,21)$ \\
\hline PSZ1G018.75+23.57 & 0.089 & $\ldots$ & $2.20 \pm 0.54$ & $0.86 \pm 0.26$ & $\ldots$ \\
\hline PSZ1G108.18-11.53 & 0.335 & $5.52 \pm 0.23(1)^{*}$ & $6.92 \pm 1.73$ & $3.99 \pm 1.19$ & $(27)$ \\
\hline PSZ1G139.61+24.20 & 0.27 & $9.22 \pm 0.23(1) *$ & $5.71 \pm 1.46$ & $3.15 \pm 0.97$ & $\ldots$ \\
\hline RXC J0142.0+2131 ${ }^{U}$ & 0.28 & $6.0 \pm 0.1(5)$ & $4.63 \pm 1.30$ & $2.29 \pm 0.79$ & . \\
\hline RX J0603.3+4214* & 0.225 & $9.12 \pm 1.90(17)$ & $9.21 \pm 1.93$ & $6.64 \pm 1.58$ & $(45,48)$ \\
\hline RXC J1314.4-2515 & 0.228 & $9.89(12)$ & $4.60 \pm 1.37$ & $2.33 \pm 0.86$ & $(50)$ \\
\hline RXJ1720.1+2638 ${ }^{U}$ & 0.164 & $9.69 \pm 0.10(6)$ & $3.98 \pm 0.89$ & $1.99 \pm 0.52$ & $\ldots$ \\
\hline Triangulum Aus. & 0.051 & $3.97 \pm 0.08(11)$ & $5.14 \pm 0.77$ & $3.13 \pm 0.47$ & .. \\
\hline $\mathrm{ZwCL}_{\mathrm{w}} 341.1+0000$ & 0.27 & $2.32 \pm 0.06(1)^{*}$ & $3.81 \pm 0.99$ & $1.72 \pm 0.54$ & $(42)$ \\
\hline $\mathrm{ZwCL5247*U}$ & 0.229 & $4.3 \pm 0.3(9)$ & $4.26 \pm 1.01$ & $2.10 \pm 0.59$ & $\ldots$ \\
\hline A 2744 & 0.307 & $14.73 \pm 0.24(5)$ & $8.76 \pm 1.84$ & $5.80 \pm 1.38$ & $(31,36)$ \\
\hline A209 & 0.206 & $7.62 \pm 0.48(5)$ & $6.31 \pm 1.32$ & $3.83 \pm 0.91$ & \\
\hline A 2163 & 0.203 & $21.95 \pm 0.33(5)$ & $15.78 \pm 2.84$ & $15.03 \pm 2.92$ & $(28)$ \\
\hline RXCJ2003.5-2323 & 0.317 & $9.17 \pm 0.09$ & $6.40 \pm 1.72$ & $3.60 \pm 1.18$ & $\ldots$ \\
\hline A520 & 0.203 & $7.81 \pm 0.21(5)$ & $5.26 \pm 1.28$ & $2.93 \pm 0.96$ & $\ldots$ \\
\hline A773 & 0.217 & $7.30 \pm 0.57(5)$ & $5.35 \pm 1.28$ & $2.97 \pm 0.84$ & $\ldots$ \\
\hline $\mathrm{A} 1758 \mathrm{~N}^{f}$ & 0.280 & $8.80 \pm 0.16(5)$ & $6.68 \pm 1.60$ & $3.96 \pm 1.12$ & $\ldots$ \\
\hline A2219 & 0.228 & $14.78 \pm 0.19(5)$ & $9.52 \pm 1.71$ & $6.97 \pm 1.35$ & $\ldots$ \\
\hline $\mathrm{A} 521^{U}$ & 0.248 & $8.28 \pm 0.07(5)$ & $5.22 \pm 1.41$ & $2.81 \pm 0.92$ & $(29,30)$ \\
\hline $\mathrm{A} 697^{U}$ & 0.282 & $13.04 \pm 0.61(5)$ & $10.78 \pm 2.26$ & $8.04 \pm 1.92$ & $\ldots$ \\
\hline $\mathrm{A} 1300^{U}$ & 0.308 & $11.47 \pm 0.37(5)$ & $8.02 \pm 1.92$ & $5.08 \pm 1.44$ & $(51)$ \\
\hline CL0016+16 & 0.541 & $15.54 \pm 0.28$ & $10.49 \pm 2.82$ & $6.31 \pm 2.06$ & $\ldots$ \\
\hline A665 & 0.182 & $8.30 \pm 0.07(5)$ & $6.32 \pm 1.32$ & $3.91 \pm 0.93$ & $\ldots$ \\
\hline A545 & 0.154 & $6.31 \pm 0.09(5)$ & $2.67 \pm 0.88$ & $1.10 \pm 0.46$ & $\ldots$ \\
\hline Coma & 0.023 & $3.39 \pm 0.03(11)$ & $2.94 \pm 0.62$ & $1.39 \pm 0.33$ & $(19,41)$ \\
\hline $\mathrm{A} 2256^{U}$ & 0.058 & $4.44 \pm 0.02(5)$ & $3.87 \pm 0.70$ & $2.04 \pm 0.40$ & $(23,25,35,46)$ \\
\hline Bullet* & 0.296 & $22.54 \pm 0.52(5)$ & $11.98 \pm 2.51$ & $9.32 \pm 2.22$ & $(38,39)$ \\
\hline $\mathrm{A} 2255^{U}$ & 0.081 & $3.31 \pm 0.03(5)$ & $3.03 \pm 0.64$ & $1.40 \pm 0.33$ & $(32,37)$ \\
\hline A2319 & 0.056 & $7.87 \pm 0.08(5)$ & $5.68 \pm 1.02$ & $3.63 \pm 0.70$ & $\ldots$ \\
\hline MACSJ0717.5+3745 & 0.548 & $24.05 \pm 0.22(5)$ & $14.68 \pm 3.51$ & $10.34 \pm 2.92$ & $(22,49)$ \\
\hline A1995 & 0.319 & $6.03 \pm 0.08(5)$ & $3.99 \pm 1.07$ & $1.78 \pm 0.58$ & . \\
\hline MACSJ1149.5+2223 ${ }^{U}$ & 0.544 & $15.50 \pm 0.29(5)$ & $10.23 \pm 2.75$ & $6.06 \pm 1.98$ & $(4)$ \\
\hline PLCKG171.9-40.7 ${ }^{U}$ & 0.270 & $11.28 \pm 0.02(13)$ & $10.31 \pm 2.16$ & $7.60 \pm 1.81$ & \\
\hline $\mathrm{A} 754^{U}$ & 0.054 & $4.75 \pm 0.033(5)$ & $4.09 \pm 0.73$ & $2.22 \pm 0.43$ & $(34)$ \\
\hline
\end{tabular}


Table 2. X-ray Luminosity values for the total sample - cont.

\begin{tabular}{|c|c|c|c|c|c|}
\hline System $^{a}$ & $z$ & $\begin{array}{c}L_{\mathrm{X} 500}[0.1-2.4 k e V]^{b} \\
\left(10^{44} \mathrm{erg} \mathrm{s}^{-1}\right)\end{array}$ & $\begin{array}{c}L_{\mathrm{Xpred}}^{\mathrm{M}}{ }^{c} \\
\left(10^{44} \mathrm{erg} \mathrm{s}^{-1}\right)\end{array}$ & $\begin{array}{c}L_{\mathrm{X} \text { pred }}^{\mathrm{G}}{ }^{d} \\
\left(10^{44} \mathrm{erg} \mathrm{s}^{-1}\right)\end{array}$ & Relics $^{e}$ \\
\hline \multicolumn{6}{|c|}{ The extra systems from Martinez Aviles et al. (2016) sample } \\
\hline A746 & 0.2323 & $3.39 \pm 1.19(18)$ & $3.78 \pm 0.93$ & $1.75 \pm 0.52$ & $(44)$ \\
\hline A1351 & 0.322 & $5.24(12)$ & $5.84 \pm 1.29$ & $3.13 \pm 0.80$ & $\ldots$ \\
\hline A1689 & 0.1832 & $13.6 \pm 1.2(9)$ & $6.77 \pm 1.35$ & $4.33 \pm 0.97$ & \\
\hline $\mathrm{A} 2034^{U}$ & 0.113 & $4.0 \pm 0.4(9)$ & $3.72 \pm 0.75$ & $1.86 \pm 0.42$ & $(40)$ \\
\hline A2254 & 0.178 & $4.79(12)$ & $3.76 \pm 0.88$ & $1.81 \pm 0.50$ & $\ldots$ \\
\hline A2294 & 0.178 & $4.05(12)$ & $4.11 \pm 0.94$ & $2.05 \pm 0.55$ & \\
\hline A3411 & 0.1687 & $2.8 \pm 0.1(15)$ & $4.60 \pm 0.96$ & $2.47 \pm 0.59$ & $(47)$ \\
\hline A3888 & 0.151 & $6.38 \pm 0.25(12)$ & $5.06 \pm 0.10$ & $2.86 \pm 0.63$ & $\ldots$ \\
\hline CIZAJ1938.3+5409 & 0.26 & $7.96(12)$ & $6.06 \pm 1.33$ & $3.47 \pm 0.88$ & $\ldots$ \\
\hline El Gordo* & 0.87 & $35.48 \pm 1.63(18)$ & $21.30 \pm 4.40$ & $13.84 \pm 3.24$ & $(33)$ \\
\hline MACSJ0553.4-3342 & 0.431 & $17(4)$ & $9.18 \pm 1.98$ & $5.64 \pm 1.40$ & $\ldots$ \\
\hline MACSJ1752.0+4440 & 0.366 & $8.0(4)$ & $6.03 \pm 1.42$ & $3.18 \pm 0.89$ & $(4)$ \\
\hline PLCKG285.0-23.7 & 0.39 & $16.91 \pm 0.27(13)$ & $8.22 \pm 1.23$ & $4.95 \pm 0.74$ & $\ldots$ \\
\hline RXCJ0107.7+5408 & 0.1066 & $2.80(12)$ & $3.69 \pm 0.80$ & $1.85 \pm 0.46$ & $(44)$ \\
\hline RXCJ0949.8+1708 & 0.38 & $11.3 \pm 2.3(9)$ & $7.89 \pm 1.93$ & $4.69 \pm 1.36$ & $\ldots$ \\
\hline RXCJ1514.9-1523 ${ }^{U}$ & 0.226 & $6.43(12)$ & $7.19 \pm 1.56$ & $4.60 \pm 1.15$ & $\cdots$ \\
\hline \multicolumn{6}{|c|}{ The upper limits systems from Cassano et al. (2013) and Kale et al. (2015) } \\
\hline A 267 & 0.230 & $5.94 \pm 0.44(5)$ & $3.51 \pm 1.04$ & $1.57 \pm 0.58$ & $\ldots$ \\
\hline A781 & 0.298 & $5.44 \pm 0.14(5)$ & $4.90 \pm 1.24$ & $2.45 \pm 0.74$ & $\ldots$ \\
\hline A1423 & 0.213 & $4.76 \pm 0.38(5)$ & $4.38 \pm 1.12$ & $2.21 \pm 0.68$ & $\ldots$ \\
\hline A1576 & 0.30 & $6.38 \pm 0.14$ & $4.75 \pm 1.20$ & $2.34 \pm 0.71$ & $\ldots$ \\
\hline A 1722 & 0.327 & $6.15(12)$ & $3.02 \pm 0.87$ & $1.17 \pm 0.41$ & $\ldots$ \\
\hline A 2485 & 0.247 & $3.07 \pm 0.07(5)$ & $3.19 \pm 0.92$ & $1.34 \pm 0.48$ & .. \\
\hline A 2537 & 0.297 & $4.54 \pm 0.07(5)$ & $4.27 \pm 1.15$ & $2.00 \pm 0.65$ & $\ldots$ \\
\hline A2631 & 0.278 & $8.62 \pm 0.70(5)$ & $6.01 \pm 1.32$ & $3.38 \pm 0.85$ & $\ldots$ \\
\hline A2645 & 0.251 & $4.13 \pm 0.4(5)$ & $2.76 \pm 0.88$ & $1.08 \pm 0.44$ & $\ldots$ \\
\hline A2697 & 0.232 & $7.29 \pm 0.41(5)$ & $4.35 \pm 1.00$ & $2.17 \pm 0.58$ & $\ldots$ \\
\hline RXCJ0439.0+0715 & 0.244 & $7.69 \pm 0.58(5)$ & $4.49 \pm 1.31$ & $2.25 \pm 0.81$ & $\ldots$ \\
\hline RXJ2228.6+2037 & 0.418 & $11.71 \pm 0.20$ & $8.36 \pm 1.84$ & $4.96 \pm 1.26$ & $\ldots$ \\
\hline ZwCL7215 & 0.2917 & $5.00 \pm 0.19(6)$ & $3.82 \pm 1.15$ & $1.71 \pm 0.64$ & $\ldots$ \\
\hline
\end{tabular}

References: X-ray References: (1 ) this work; (2) Albert et al. (2017); (3) Bîrzan et al. (2017); (4) Bonafede et al. (2012); (5) Cassano et al. (2013); (6) Giles et al. (2017); (7) Hoang et al. (2017); (8) Knowles et al. (2016); (9) Mantz et al. (2010); (10) Ogrean et al. (2015); (11) O'Hara et al. (2006); (12) Piffaretti et al. (2011); (13) Planck Collaboration et al. (2011); (14) Vikhlinin et al. (2009) (15) van Weeren et al. (2013) (16) Wilber et al. (2018); (17) Wu et al. (1999); (18) Yuan et al. (2015). Relics references: (19) Andernach et al. (1984) (20) Bagchi et al. (2011) (21) Bonafede et al. (2014a) (22) Bonafede et al. (2018) (23) Brentjens (2008) (24) Cantwell et al. (2016) (25) Clarke \& Ensslin (2006) (26) Cuciti et al. (2018) (27) de Gasperin et al. (2015) (28) Feretti et al. (2001) (29) Giacintucci et al. (2006) (30) Giacintucci et al. (2008) (31) Govoni et al. (2001) (32) Govoni et al. (2005) (33) Lindner et al. (2014) (34) Macario et al. (2011) (35) Owen et al. (2014) (36) Pearce et al. (2017) (37) Pizzo \& de Bruyn (2009) (38) Shimwell et al. (2014) (39) Shimwell et al. (2015) (40) Shimwell et al. (2016) (41) Thierbach et al. (2003) (42) van Weeren et al. (2009) (43) van Weeren et al. (2010) (44) van Weeren et al. (2011) (45) van Weeren et al. (2012a) (46) van Weeren et al. (2012b) (47) van Weeren et al. (2013) (48) van Weeren et al. (2016a) (49) van Weeren et al. (2017b) (50) Venturi et al. (2007) (51) Venturi et al. (2013)

${ }^{a}$ Radio halo systems taken from the literature including those in the Cassano et al. (2013) and Martinez Aviles et al. (2016) samples, and the systems with upper limits. The asterisk marks systems with alternative names: Bullet (1E 0657-56), El Gordo (ACT-CL J0102-4915), with the others listed in Table 1. And, as in Table 1. The 'U' marks the systems with steep-spectrum RHs $(\alpha>1.5)$.

${ }^{b} \mathrm{X}$-ray luminosity between $0.1-2.4 \mathrm{keV}$ within $\mathrm{R}_{500}$, except for CL1446+26, where only the bolometric X-ray luminosity was available in the literature (Wu et al. 1999); and for the systems from O'Hara et al. (2006) and Vikhlinin et al. (2009), where the 0.5-2.0 keV energy band was used. For the systems marked with asterisk, since there were no available X-ray luminosities in the literature, we reduced the Chandra X-ray data (ObsIDs $15139,17490,17213)$ ourselves, following the same reduction scheme described in Section 2.3.

${ }^{c}$ The predicted X-ray luminosity between $0.1-2.4 \mathrm{keV}$ within $\mathrm{R}_{500}$ using the L-M scaling relations of Mantz et al. (2010).

${ }^{d}$ The predicted X-ray luminosity between $0.1-2.4 \mathrm{keV}$ within $\mathrm{R}_{500}$ using the L-M scaling relations of Giles et al. (2017).

${ }^{e}$ The presence of relics (radio shocks) from literature.

$f$ There is also a RH in A1758S (Botteon et al. 2018). 


\section{ACKNOWLEDGEMENTS}

The scientific results reported in this article are based on data obtained with the International LOFAR Telescope (ILT), and archive data from Chandra Data Archive and XMM-Newton archive. LOFAR (van Haarlem et al. 2013) is the Low Frequency Array designed and constructed by ASTRON. It has observing, data processing, and data storage facilities in several countries, that are owned by various parties (each with their own funding sources), and that are collectively operated by the ILT foundation under a joint scientific policy. The ILT resources have benefitted from the following recent major funding sources: CNRS-INSU, Observatoire de Paris and Universit d'Orlans, France; BMBF, MIWF-NRW, MPG, Germany; Science Foundation Ireland (SFI), Department of Business, Enterprise and Innovation (DBEI), Ireland; NWO, The Netherlands; The Science and Technology Facilities Council, UK.

The LOFAR reduction was done using PREFACTOR and FACTOR packages, and the X-ray data reduction has made using CIAO package provided by Chandra X-ray Center $(\mathrm{CXC})$, and SAS package for XMM-Newton data. The LOFAR group in Leiden is supported by the ERC Advanced Investigator program New-Clusters 321271. The authors thank the referee for the constructive comments, which improve the paper significantly.

\section{REFERENCES}

Albert J. G., Sifón C., Stroe A., Mernier F., Intema H. T., Röttgering H. J. A., Brunetti G., 2017, A\&A, 607, A4

Alexander D. M., Hickox R. C., 2012, NewAR, 56, 93

Allen S. W., Evrard A. E., Mantz A. B., 2011, ARAA, 49, 409

Allen S. W., Schmidt R. W., Fabian A. C., Ebeling H., 2003, MNRAS, 342, 287

Andernach H., Feretti L., Giovannini G., 1984, A\&A, 133, 252

Andrade-Santos F. et al., 2017, ApJ, 843, 76

Arnaud K. A., 1996, in Astronomical Society of the Pacific Conference Series, Vol. 101, Astronomical Data Analysis Software and Systems V, G. H. Jacoby \& J. Barnes, ed., p. 17

Bacchi M., Feretti L., Giovannini G., Govoni F., 2003, A\&A, 400, 465

Bagchi J. et al., 2011, ApJ, 736, L8

Basu K., 2012, MNRAS, 421, L112

Benson A. J., Bower R. G., Frenk C. S., Lacey C. G., Baugh C. M., Cole S., 2003, ApJ, 599, 38

Bernardi G. et al., 2016, MNRAS, 456, 1259

Bîrzan L., Rafferty D. A., Brüggen M., Intema H. T., 2017, MNRAS, 471, 1766

Bîrzan L., Rafferty D. A., McNamara B. R., Wise M. W., Nulsen P. E. J., 2004, ApJ, 607, 800

Bîrzan L., Rafferty D. A., Nulsen P. E. J., McNamara B. R., Röttgering H. J. A., Wise M. W., Mittal R., 2012, MNRAS, 427, 3468

Böhringer H. et al., 2000, ApJS, 129, 435

Bonafede A. et al., 2018, MNRAS, 478, 2927

Bonafede A. et al., 2012, MNRAS, 426, 40
Bonafede A., Intema H. T., Brüggen M., Girardi M., Nonino M., Kantharia N., van Weeren R. J., Röttgering H. J. A., 2014a, ApJ, 785, 1

Bonafede A. et al., 2014b, MNRAS, 444, L44

Boschin W., Barrena R., Girardi M., 2009, A\&A, 495, 15

Botteon A., Gastaldello F., Brunetti G., Kale R., 2016, MNRAS, 463, 1534

Botteon A. et al., 2018, MNRAS, 478, 885

Bourdin H., Mazzotta P., Markevitch M., Giacintucci S., Brunetti G., 2013, ApJ, 764, 82

Brentjens M. A., 2008, A\&A, 489, 69

Brown S., Duesterhoeft J., Rudnick L., 2011, ApJ, 727, L25

Brüggen M., Bykov A., Ryu D., Röttgering H., 2012, Space

Science Reviews, 166, 187

Brunetti G., Cassano R., Dolag K., Setti G., 2009, A\&A, 507,661

Brunetti G., Jones T. W., 2014, International Journal of Modern Physics D, 23, 1430007

Brunetti G., Lazarian A., 2011, MNRAS, 412, 817

Brunetti G., Lazarian A., 2016, MNRAS, 458, 2584

Brunetti G., Setti G., Feretti L., Giovannini G., 2001, MNRAS, 320, 365

Brunetti G., Venturi T., Dallacasa D., Cassano R., Dolag K., Giacintucci S., Setti G., 2007, ApJ, 670, L5

Brunetti G., Zimmer S., Zandanel F., 2017, MNRAS, 472, 1506

Burns J. O., Hallman E. J., Gantner B., Motl P. M., Norman M. L., 2008, ApJ, 675, 1125

Canning R. E. A. et al., 2017, MNRAS, 464, 2896

Cantwell T. M., Scaife A. M. M., Oozeer N., Wen Z. L., Han J. L., 2016, MNRAS, 458, 1803

Cassano R., Brunetti G., Norris R. P., Röttgering H. J. A., Johnston-Hollitt M., Trasatti M., 2012, A\&A, 548, A100

Cassano R., Brunetti G., Röttgering H. J. A., Brüggen M., 2010a, A\&A, 509, A68

Cassano R., Brunetti G., Setti G., 2006, MNRAS, 369, 1577

Cassano R., Brunetti G., Setti G., Govoni F., Dolag K., 2007, MNRAS, 378, 1565

Cassano R. et al., 2013, ApJ, 777, 141

Cassano R., Ettori S., Giacintucci S., Brunetti G., Marke-

vitch M., Venturi T., Gitti M., 2010b, ApJ, 721, L82

Clarke T. E., Ensslin T. A., 2006, AJ, 131, 2900

Clowe D., Bradač M., Gonzalez A. H., Markevitch M., Randall S. W., Jones C., Zaritsky D., 2006, ApJ, 648, L109

Clowe D., Gonzalez A., Markevitch M., 2004, ApJ, 604, 596

Clowe D., Markevitch M., Bradač M., Gonzalez A. H., Chung S. M., Massey R., Zaritsky D., 2012, ApJ, 758, 128

Connor T., Donahue M., Sun M., Hoekstra H., Mahdavi A., Conselice C. J., McNamara B., 2014, ApJ, 794, 48

Cooray A. R., Grego L., Holzapfel W. L., Joy M., Carlstrom J. E., 1998, AJ, 115, 1388

Cuciti V., Brunetti G., van Weeren R., Bonafede A., Dallacasa D., Cassano R., Venturi T., Kale R., 2018, A\&A, 609, A61

Cuciti V., Cassano R., Brunetti G., Dallacasa D., Kale R., Ettori S., Venturi T., 2015, A\&A, 580, A97

Dahle H., Kaiser N., Irgens R. J., Lilje P. B., Maddox S. J., 2002, ApJS, 139, 313

Dahle H., Pedersen K., Lilje P. B., Maddox S. J., Kaiser N., 2003, ApJ, 591, 662

Dasadia S. et al., 2016, ApJ, 820, L20 
de Gasperin F., Intema H. T., van Weeren R. J., Dawson W. A., Golovich N., Wittman D., Bonafede A., Brüggen M., 2015, MNRAS, 453, 3483

Dickey J. M., Lockman F. J., 1990, ARAA, 28, 215

Donnert J., Dolag K., Brunetti G., Cassano R., 2013, MNRAS, 429, 3564

Duchesne S. W., Johnston-Hollitt M., Offringa A. R., Pratt G. W., Zheng Q., Dehghan S., 2017, ArXiv e-prints

Eckert D., Gaspari M., Vazza F., Gastaldello F., Tramacere A., Zimmer S., Ettori S., Paltani S., 2017, ApJ, 843, L29

Eckert D., Jauzac M., Vazza F., Owers M. S., Kneib J.-P., Tchernin C., Intema H., Knowles K., 2016, MNRAS, 461, 1302

Ensslin T. A., Biermann P. L., Klein U., Kohle S., 1998, A\&A, 332, 395

Fabian A. C., 2012, ARAA, 50, 455

Farnsworth D., Rudnick L., Brown S., Brunetti G., 2013, ApJ, 779, 189

Feretti L., Fusco-Femiano R., Giovannini G., Govoni F., 2001, A\&A, 373, 106

Feretti L., Giovannini G., Govoni F., Murgia M., 2012, aapr, 20, 54

Feretti L., Orrù E., Brunetti G., Giovannini G., Kassim N., Setti G., 2004, A\&A, 423, 111

Ferrari C. et al., 2011, A\&A, 534, L12

Finoguenov A., Sarazin C. L., Nakazawa K., Wik D. R., Clarke T. E., 2010, ApJ, 715, 1143

Giacintucci S., Dallacasa D., Venturi T., Brunetti G., Cassano R., Markevitch M., Athreya R. M., 2011, A\&A, 534, A57

Giacintucci S., Venturi T., Bardelli S., Brunetti G., Cassano R., Dallacasa D., 2006, NewA, 11, 437

Giacintucci S. et al., 2005, A\&A, 440, 867

Giacintucci S., Venturi T., Brunetti G., Dallacasa D., Mazzotta P., Cassano R., Bardelli S., Zucca E., 2009, A\&A, 505,45

Giacintucci S. et al., 2008, A\&A, 486, 347

Giles P. A. et al., 2017, MNRAS, 465, 858

Giovannini G., Bonafede A., Feretti L., Govoni F., Murgia M., 2010, A\&A, 511, L5

Giovannini G., Bonafede A., Feretti L., Govoni F., Murgia M., Ferrari F., Monti G., 2009, A\&A, 507, 1257

Giovannini G., Feretti L., 2000, New Astronomy, 5, 335

Girardi M. et al., 2016, MNRAS, 456, 2829

Golovich N. et al., 2018, ArXiv e-prints

Govoni F., Enßlin T. A., Feretti L., Giovannini G., 2001, A\&A, 369, 441

Govoni F., Ferrari C., Feretti L., Vacca V., Murgia M., Giovannini G., Perley R., Benoist C., 2012, A\&A, 545, A74

Govoni F., Murgia M., Feretti L., Giovannini G., Dallacasa D., Taylor G. B., 2005, A\&A, 430, L5

Ha J.-H., Ryu D., Kang H., 2018, ApJ, 857, 26

Hahn O., Martizzi D., Wu H.-Y., Evrard A. E., Teyssier R., Wechsler R. H., 2017, MNRAS, 470, 166

Harvey D., Massey R., Kitching T., Taylor A., Tittley E., 2015, Science, 347, 1462

Hasselfield M. et al., 2013, JCAP, 7, 008

Hlavacek-Larrondo J. et al., 2018, MNRAS, 475, 2743

Hoang D. N. et al., 2017, MNRAS, 471, 1107

Hoang D. N. et al., 2019a, A\&A, 622, A20

Hoang D. N. et al., 2019b, A\&A, 622, A21
Hoeft M., Nuza S. E., Gottlöber S., van Weeren R. J., Röttgering H. J. A., Brüggen M., 2011, Journal of Astrophysics and Astronomy, 32, 509

Intema H. T., Jagannathan P., Mooley K. P., Frail D. A., 2017, A\&A, 598, A78

Irgens R. J., Lilje P. B., Dahle H., Maddox S. J., 2002, ApJ, 579,227

Jauzac M. et al., 2016, MNRAS, 463, 3876

Jee M. J., Dawson W. A., Stroe A., Wittman D., van Weeren R. J., Brüggen M., Bradač M., Röttgering H., 2016, ApJ, 817, 179

Jee M. J., Hughes J. P., Menanteau F., Sifón C., Mandelbaum R., Barrientos L. F., Infante L., Ng K. Y., 2014 , ApJ, 785, 20

Jee M. J., Mahdavi A., Hoekstra H., Babul A., Dalcanton J. J., Carroll P., Capak P., 2012, ApJ, 747, 96

Kale R., 2018, arXiv e-prints

Kale R., Venturi T., Cassano R., Giacintucci S., Bardelli S., Dallacasa D., Zucca E., 2015, A\&A, 581, A23

Kale R., Venturi T., Giacintucci S., Dallacasa D., Cassano R., Brunetti G., Macario G., Athreya R., 2013, A\&A, 557, A99

Kang H., Ryu D., Jones T. W., 2012, ApJ, 756, 97

Kim K.-T., Kronberg P. P., Dewdney P. E., Landecker T. L., 1990, ApJ, 355, 29

Knowles K. et al., 2016, MNRAS, 459, 4240

Kravtsov A. V., Borgani S., 2012, ARAA, 50, 353

Lindner R. R. et al., 2014, ApJ, 786, 49

Macario G., Markevitch M., Giacintucci S., Brunetti G., Venturi T., Murray S. S., 2011, ApJ, 728, 82

Macario G. et al., 2013, A\&A, 551, A141

Mahdavi A., Hoekstra H., Babul A., Balam D. D., Capak P. L., 2007, ApJ, 668, 806

Mahdavi A., Hoekstra H., Babul A., Bildfell C., Jeltema T., Henry J. P., 2013, ApJ, 767, 116

Mandal S. et al., 2019, A\&A, 622, A22

Mantz A., Allen S. W., Ebeling H., Rapetti D., DrlicaWagner A., 2010, MNRAS, 406, 1773

Mantz A. B., Allen S. W., Morris R. G., Schmidt R. W., 2016, MNRAS, 456, 4020

Markevitch M., Gonzalez A. H., Clowe D., Vikhlinin A., Forman W., Jones C., Murray S., Tucker W., 2004, ApJ, 606,819

Markevitch M., Gonzalez A. H., David L., Vikhlinin A., Murray S., Forman W., Jones C., Tucker W., 2002, ApJ, 567, L27

Markevitch M., Govoni F., Brunetti G., Jerius D., 2005, ApJ, 627, 733

Martinez Aviles G. et al., 2016, A\&A, 595, A116

McNamara B. R., Nulsen P. E. J., 2007, ARAA, 45, 117

Medezinski E., Battaglia N., Coupon J., Cen R., Gaspari M., Strauss M. A., Spergel D. N., 2017, ApJ, 836, 54

Mernier F. et al., 2017, A\&A, 603, A80

Merten J. et al., 2011, MNRAS, 417, 333

Murgia M., Govoni F., Feretti L., Giovannini G., 2010, A\&A, 509, A86+

Nuza S. E., Hoeft M., van Weeren R. J., Gottlöber S., Yepes G., 2012, MNRAS, 420, 2006

Ogrean G. A. et al., 2015, ApJ, 812, 153

O'Hara T. B., Mohr J. J., Bialek J. J., Evrard A. E., 2006, ApJ, 639, 64

Okabe N., Umetsu K., 2008, PASJ, 60, 345 
Orrú E., Murgia M., Feretti L., Govoni F., Brunetti G., Giovannini G., Girardi M., Setti G., 2007, A\&A, 467, 943 Owen F., Morrison G., Voges W., 1999, in Diffuse Thermal and Relativistic Plasma in Galaxy Clusters, Boehringer H., Feretti L., Schuecker P., eds., p. 9

Owen F. N., Rudnick L., Eilek J., Rau U., Bhatnagar S., Kogan L., 2014, ApJ, 794, 24

Parekh V., Dwarakanath K. S., Kale R., Intema H., 2017, MNRAS, 464, 2752

Parrish I. J., Quataert E., Sharma P., 2010, ApJ, 712, L194

Pearce C. J. J. et al., 2017, ApJ, 845, 81

Peel A., Lanusse F., Starck J.-L., 2017, ApJ, 847, 23

Petrosian V., 2001, ApJ, 557, 560

Pfrommer C., Chang P., Broderick A. E., 2012, ApJ, 752, 24

Pfrommer C., Enßlin T. A., Springel V., 2008, MNRAS, 385,1211

Piffaretti R., Arnaud M., Pratt G. W., Pointecouteau E., Melin J.-B., 2011, A\&A, 534, A109

Pinzke A., Oh S. P., Pfrommer C., 2013, MNRAS, 435, 1061

Pinzke A., Oh S. P., Pfrommer C., 2017, MNRAS, 465, 4800

Pizzo R. F., de Bruyn A. G., 2009, A\&A, 507, 639

Planck Collaboration et al., 2014, A\&A, 571, 29

Planck Collaboration et al., 2015, VizieR Online Data Catalog, 358

Planck Collaboration et al., 2016, A\&A, 594, A27

Planck Collaboration et al., 2011, A\&A, 536, A9

Poole G. B., Babul A., McCarthy I. G., Sanderson A. J. R., Fardal M. A., 2008, MNRAS, 391, 1163

Pratt G. W., Croston J. H., Arnaud M., Böhringer H., 2009, A\&A, 498, 361

Press W. H., Schechter P., 1974, ApJ, 187, 425

Randall S. W., Sarazin C. L., Ricker P. M., 2002, ApJ, 577, 579

Rasia E. et al., 2015, ApJ, 813, L17

Reiprich T. H., Böhringer H., 2002, ApJ, 567, 716

Ricker P. M., Sarazin C. L., 2001, ApJ, 561, 621

Ritchie B. W., Thomas P. A., 2002, MNRAS, 329, 675

Rossetti M., Gastaldello F., Eckert D., Della Torre M., Pantiri G., Cazzoletti P., Molendi S., 2017, MNRAS, 468, 1917

Rudnick L., Lemmerman J. A., 2009, ApJ, 697, 1341

Russell H. R., Fabian A. C., Sanders J. S., Johnstone R. M., Blundell K. M., Brandt W. N., Crawford C. S., 2010, MNRAS, 402, 1561

Sanders J. S., Fabian A. C., 2007, MNRAS, 381, 1381

Sarazin C. L., Finoguenov A., Wik D. R., Clarke T. E., 2016, ArXiv e-prints

Savini F. et al., 2019, A\&A, 622, A24

Savini F. et al., 2018, MNRAS, 478, 2234

Scaife A. M. M., Heald G. H., 2012, MNRAS, 423, L30

Scannapieco E., Oh S. P., 2004, ApJ, 608, 62

Shakouri S., Johnston-Hollitt M., Pratt G. W., 2016, MNRAS, 459, 2525

Shimwell T. W., Brown S., Feain I. J., Feretti L., Gaensler B. M., Lage C., 2014, MNRAS, 440, 2901

Shimwell T. W. et al., 2016, MNRAS, 459, 277

Shimwell T. W., Markevitch M., Brown S., Feretti L., Gaensler B. M., Johnston-Hollitt M., Lage C., Srinivasan R., 2015, MNRAS, 449, 1486

Shimwell T. W. et al., 2017, A\&A, 598, A104
Smith R. K., Brickhouse N. S., Liedahl D. A., Raymond J. C., 2001, ApJ, 556, L91

Sommer M. W., Basu K., Intema H., Pacaud F., Bonafede A., Babul A., Bertoldi F., 2017, MNRAS, 466, 996

Springel V., Frenk C. S., White S. D. M., 2006, Nature, 440, 1137

Storm E., Jeltema T. E., Rudnick L., 2015, MNRAS, 448, 2495

Sun M., 2009, ApJ, 704, 1586

Thierbach M., Klein U., Wielebinski R., 2003, A\&A, 397, 53

Vacca V., Feretti L., Giovannini G., Govoni F., Murgia M., Perley R. A., Clarke T. E., 2014, A\&A, 561, A52

van Haarlem M. P. et al., 2013, A\&A, 556, A2

van Weeren R. J. et al., 2017a, Nature Astronomy, 1, 0005 van Weeren R. J., Brüggen M., Röttgering H. J. A., Hoeft M., Nuza S. E., Intema H. T., 2011, A\&A, 533, A35

van Weeren R. J. et al., 2016a, ApJ, 818, 204

van Weeren R. J., de Gasperin F., Akamatsu H., Brüggen M., Feretti L., Kang H., Stroe A., Zandanel F., 2019, Space Science Rewiews, 215, 16

van Weeren R. J. et al., 2013, ApJ, 769, 101

van Weeren R. J. et al., 2017b, ApJ, 835, 197

van Weeren R. J. et al., 2009, A\&A, 506, 1083

van Weeren R. J., Röttgering H. J. A., Brüggen M., Hoeft M., 2010, Science, 330, 347

van Weeren R. J., Röttgering H. J. A., Intema H. T., Rudnick L., Brüggen M., Hoeft M., Oonk J. B. R., 2012a, A\&A, 546, A124

van Weeren R. J. et al., 2012b, A\&A, 543, A43

van Weeren R. J. et al., 2016b, ApJS, 223, 2

Vazza F., Brüggen M., Gheller C., Brunetti G., 2012, MNRAS, 421, 3375

Venturi T., Bardelli S., Dallacasa D., Brunetti G., Giacintucci S., Hunstead R. W., Morganti R., 2003, A\&A, 402, 913

Venturi T., Giacintucci S., Brunetti G., Cassano R., Bardelli S., Dallacasa D., Setti G., 2007, A\&A, 463, 937

Venturi T., Giacintucci S., Dallacasa D., Cassano R., Brunetti G., Bardelli S., Setti G., 2008, A\&A, 484, 327

Venturi T., Giacintucci S., Dallacasa D., Cassano R., Brunetti G., Macario G., Athreya R., 2013, A\&A, 551, A24

Venturi T. et al., 2017, A\&A, 603, A125

Vikhlinin A. et al., 2009, ApJ, 692, 1033

Voit G. M., Kay S. T., Bryan G. L., 2005, MNRAS, 364, 909

Wang Q. H. S., Markevitch M., Giacintucci S., 2016, ApJ, 833, 99

Wilber A. et al., 2018, MNRAS, 473, 3536

Wittman D., Golovich N., Dawson W. A., 2018, ApJ, 869, 104

Wu X.-P., Xue Y.-J., Fang L.-Z., 1999, ApJ, 524, 22

Yuan Z. S., Han J. L., Wen Z. L., 2015, ApJ, 813, 77

Zandanel F., Pfrommer C., Prada F., 2014, MNRAS, 438, 124 